\title{
BEHAVIOR OF MERCURY, LEAD, CESIUM, AND URANYL IONS ON FOUR SRS SOILS (U)
}

by J. P. Bibler, et al.

WSRC-RP--92-326

Westinghouse Savannah River Company

Savannah River Site

DE93 003378

Aiken, South Carolina 29808

D. B. Marson

This paper was prepared in connection with work done under Contract No. DE-AC09-89SR1803.5 with the U. S. Department of Energy. By acceptance of this paper, the publisher and/or recipient acknowledges the U. S. Government's right to retain a nonexclusive, royalty-free license in and to any copyright covering this paper, along with the right to reproduce and to authorize others to reproduce all or part of the copyrighted paper.

\section{MASIER}




\section{DISCLAIMER}

This report was prepared as an account of work sponsored by an agency of the United States Government. Neither the United States Government nor any agency thereof, nor any of their employees, makes any warranty, express or implied, or assumes any legal liability or responsibility for the accuracy, completeness, or usefulness of any information, apparatus, product, or process disclosed, or represents that its use would not infringe privately owned rights. Reference herein to any specific commercial product, process, or service by trade name, trademark, manufacturer, or otherwise does not necessarily constitute or imply its endorsement, recommendation, or favoring by the United States Government or any agency thereof. The views and opinions of authors expressed herein do not necessarily state or reflect those of the United States Government or any agency thereof.

This report has been reproduced directly from the best available copy.

Available to DOE and DOE contractors from the Office of Scientific and Technical Information, P.O. Box 62, Oak Ridge, TN 37831; prices available from (615) 576-8401, FTS 626-8401.

Available to the public from the National Technical Information Service, U.S. Department of Commerce, 5285 Port Royal Rd., Springfield, VA 22161. 
WESTIXGEOOSE SAVAMARE RIVER COMPANT SAVAMIAB RIVER IAABORATORY

TO: D.I. FISE, 773-A
WSRC-RP-92-326

Reywords:

Soil Decontamination, Heavy Metals. Mercury Decontamination, Lead Decontamination, C8-137 Decontamination

March 20, 1992

FROY J.P PIBLER, 773-A, and D.B. MARSOM

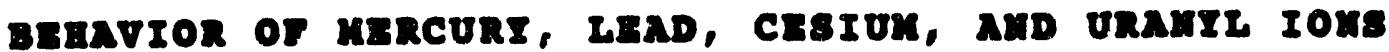

on Four sRs sorzs (U)

\section{$1.0 \operatorname{son} \sin x$}

Samples of four sRs solls were teated for sorption behavior with $\mathrm{Bg}^{2+}, \mathrm{Pb}^{2+}, \mathrm{OO}_{2}{ }^{2+}$, and $\mathrm{Cs}^{+}$tons. The purpose of the study was to determine the selectivity of the different solls for these fons alone and in the presence of the competing cations, $\mathrm{B}^{+}$and $\mathrm{Ca}^{2+}$. Distribution constants, Kd's, for the test lons in various solutions have been determined for the four solls. In general, sorption by all of the solls appeared to be nore complex than a imple lon exchange or adrorption procese. In particular, the presence of organic matter in 0011 increased the capacity of the soll due to ite chelating ability. Sinilar solie did not react similarly toward each metal cation. The "soft" watal lons, $\mathrm{Bg}^{2+}$ and $\mathrm{Pb}^{2+}$, were sorbed by silica sand in the absence of clay. whereas the "hard" $\mathrm{Cs}^{+}$and $\mathrm{UO}_{2}{ }^{2+}$ lons were not. Competition with $\mathrm{B}^{+}$and $\mathrm{Ca}^{2+}$ in reacting solutions reduced the ability of each soll to sorb the test metal lons. Sorption reactions were not easily reversible, however, indlcating that the use of $\mathrm{kd}$ values for modeling desorption is too aimplistic an approach. zeta potential, pE, and particle size also dictate the sorption behavior of solls.

\subsection{IMTnODOCrion}

To date, the mothod of managing contaminated soll at the savannah River site (SRs) remains loading in a B-25 and tranefer of the solls to the burlal ground. The volune of contaninated and suspect soll that must be dealt with in this way on an annual basis is large and generates large costs, estinated at $\$ 51 / \mathrm{ft}^{3}$. Several new technologies, such as soll washing, leaching, and electrolytic migration, are being considered to mininize costs and the amount of soll that must be dug up and moved. scouting experiments designed to toet the feasibility of som of those now technologies have shown that the composition of the soll. soll swelling, and the nature of the ion impurities of concern are very interdependent relative to sorption and desorption parameters. Thic study represents an initial attempt to guantify the behavior 
of four common contaminant lons, $\mathrm{Bg}^{2+}, \mathrm{Pb}^{2+}, \mathrm{VO}_{2}{ }^{2+}$, and $\mathrm{Cs}^{+}$, with soils from four different site locations relative to the selectivity of those solls for the metal ions and the ability of the soils to desorb them.

\subsection{TzPERIMEmInx}

Four soil samples were obtained from larger samples that had already been collected on site for other studies. Two of these, TF1 and TF2, were soils taken from two different locations in the SRs burlal ground. A third, the Tix soll, was collected at a seep line near the old Tix basin. The fourth, BTF, was collected near the F/B Bffluent Treatment Facility. Soll samples were air-dried before welghing. TF1, TF2, and Tix so1la were proceseed on sieves and the 40-60 mesh and 80-100 meah fractions wero collected for use in this seudy. Raolinite was separated from sand in these samples by making a slurry of the soll and naking several decantations of the flner kaolinite particles. The heavier sand particles remained behind in the elurry vessel. The BIF soll was not sieved because it had a residual activity of $4000 \mathrm{dpm} / \mathrm{p} / \mathrm{h}$ at one inch and would have contaninated the sleves. All work with the BIF soil was done in a radioactive hood. Although overy offort was made to try to generate BTF sapples that appeared as physically sintlar to each other as poseible, wore variablility is to be expected in these relative to the other soll eapples that were sieved.

Samples of each soil were sent for characterization by X-ray diffraction.

\subsection{Bulk Density Deternination}

The bulk dens1ty. $P_{B}$, is defined as the mase of dry material per unit volume of wetted material. Air-dried soll samples of 40-60 mesh TF1, TF2, and THX so11s and unsieved BTF soll were weighed and placed in a $10 \mathrm{~mL}$ graduate cylinder. The solutions uned for deterining distribution constants (Section 3.2) were added to the cylinder such that the soil was thoroughly wotted and an excess of about 5 in of solution remained above the soll sample. The soll was then allowed to stand in the solution for 24 hours. after which time the volume of the soll sample was nosured.

\subsection{Dletribution Constant, ra, Doternination of sorption}

Welghed samples of afr-drled soll were shaken for 48 hours with a magsured amount of solution. Solutions used weres

$$
\begin{aligned}
& \text { - } \mathrm{pb}^{2+}-125 \mathrm{ppm}, 100 \mathrm{ppm}, 75 \mathrm{ppa}, 50 \mathrm{ppa} \text { as } \mathrm{Pb}\left(\mathrm{NO}_{3}\right)_{2} \\
& \mathrm{pb}^{2+} \text { asd } \mathrm{a}^{+}-100 \mathrm{pp} \mathrm{pb}^{2+} \text { at } \mathrm{pB} 1,2,3,4
\end{aligned}
$$


$\mathrm{Pb}^{2+}$ In $2 \mathrm{M} \mathrm{Ca}^{2+}-125 \mathrm{ppm} \mathrm{Pb}^{2+}, 100 \mathrm{ppm} \mathrm{Pb}^{2+}, 75 \mathrm{ppm}^{2+} \mathrm{Pb}^{2+}$ $50 \mathrm{ppm} \mathrm{Pb}^{2+}$ all in $2 \mathrm{M} \mathrm{Ca}\left(\mathrm{NO}_{3}\right)$

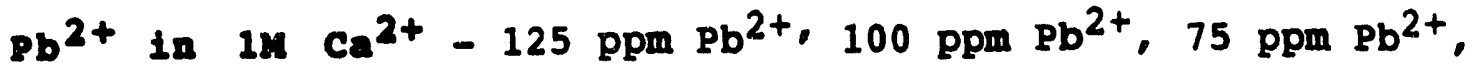
50 ppm $\mathrm{Pb}^{2+}$ all in $1 \mathrm{M} \mathrm{Ca}\left(\mathrm{NO}_{3}\right)_{2}$

- $\mathrm{Eg}^{2+}-1 \mathrm{ppm}, 2 \mathrm{ppm}, 3 \mathrm{ppm}, 5 \mathrm{ppm}$ as $\mathrm{Hg}\left(\mathrm{NO}_{3}\right)_{2}$ $\mathrm{Bg}^{2+}$ and $\mathrm{at}^{+}-5 \mathrm{ppm} \mathrm{Bg}^{2+}$ at $\mathrm{pB} 1,2,3,4$ $\mathrm{Bg}^{2+}$ in $2 \mathrm{M} \mathrm{Ca}^{2+}-1$ ppm $\mathrm{Bg}^{2+}, 2 \mathrm{ppm} \mathrm{Bg}^{2+}, 3 \mathrm{ppm} \mathrm{Bg}^{2+}$.

$5 \mathrm{ppm} \mathrm{Eg}^{2+}$ all in $2 \mathrm{M} \mathrm{Ca}\left(\mathrm{NO}_{3}\right)_{2}$



$5 \mathrm{ppm} \mathrm{Eg}^{2+}$ all in $1 \mathrm{M} \mathrm{Ca}\left(\mathrm{NO}_{3}\right)_{2}$ - $\mathrm{Cs}^{+}-1 \mathrm{M}, 0.1 \mathrm{M}, 0.05 \mathrm{M}, 0.01 \mathrm{M} \mathrm{CsHO}_{3}$ (non-radioactive) and $2.80 \mathrm{~B}-3 \mu \mathrm{CI} / \mathrm{mr} \mathrm{Cs}-137$ as $\mathrm{Cs}\left(\mathrm{HO}_{3}\right)_{2}$

- $\mathrm{UO}_{2}{ }^{2+}-2,245 \mathrm{ppm} \mathrm{O}$ as $\mathrm{UO}_{2}\left(\mathrm{NO}_{3}\right)_{2}$

The soll was then separated from the equilibrium solution by filtration and the filtrate was sent for analyalo to deterine equilibrium concentrations of lons of interest. These were compared with concentrations of the san lons in the feed solutions. Id values, which have units of $\mathrm{mr} / \mathrm{g}$. were calculated from the following relationship.

$$
\mathrm{Rd}=[(\mathrm{CO}-\mathrm{C}) / \mathrm{C}][\mathrm{V} / \mathrm{m}]
$$

$$
\begin{aligned}
& \text { where Co }=\text { initial concentration of the ton } \\
& C=\text { equilibrium concentration of the ion } \\
& \vec{V}=\text { mr of solution used } \\
& m=\text { grams of dry soil }
\end{aligned}
$$

Distribution constants for silica sand were determined in the same manner using $\mathrm{Cs}^{+}, \mathrm{UO}_{2}{ }^{2+}, \mathrm{Bg}^{2+}$, and $\mathrm{Pb}^{2+}$ solutions.

\subsection{Deserption studies}

Weighed samples of air-drled solls were soaked for 48 houre in measured volumes of solutions containing knom concentrations of $\mathrm{Ag}^{2+}, \mathrm{Pb}^{2+}, \mathrm{Cs}^{+}$, and $\mathrm{UO}_{2}{ }^{2+}$ ions. The soils were separated from the liquor by filtraton. The precipitate was washed with $10 \mathrm{ml}$ of deionized water and the wash was mixed in with the equilibrium liquor: This mixture was analyzed for the reasining concentration of the ion of interest and a correction was made for the additional volum of water added. The amount of Ion sorbed by the soll was determined by difference between the concentrations in the original solution before contacting the soll and the volum- 
corrected equilibrium solution. The milligrams of ion of interest per gram of soil was calculated.

The air-dried, contaminated soil was then divided into smaller, weighed samples which were shaken vigorously for 48 hours with measured volumes of $\mathrm{O} .1 \mathrm{M} \mathrm{BNO}_{3}$ or $1 \mathrm{M} \mathrm{Ca}\left(\mathrm{NO}_{3}\right)_{2}$. At the end of that time, the soll was again separated from the liquid by filtration and the filtrate was analyzed for the concentration of the ion of interest in order to quantify the amount of ion desorbed in the process.

\subsection{Doternination if Organio Content of THX 8011}

The physical appeara: je of the TwX 8011, whtch was black and contained leaves and ocher organic matter that nade it amorphous, was different from the other three 8011s. The TP1, TF2, and ETP soils were reddish brown, granular materials. A weighed sample of 40-60 mesh THX soll was heated 2 houre at $90^{\circ} \mathrm{C}$ in a crucible in an oven to drive off any interstitial water. After weighing to constant weight, the soll was transferred to a smaller, weighed crucible and then heated to $150^{\circ} \mathrm{C}$ to drive off bonnd water. The sample was brought to constant welght and heated again to $600^{\circ} \mathrm{C}$ for 16 hours to burn away organic matter. The difference between the welghts of the sample heated to $150^{\circ} \mathrm{C}$ and heated to $600^{\circ} \mathrm{C}$ was used to determine the per cent organic matter associated with the Tix soll.

\subsection{REsurTs}

\subsection{General soll Characterization}

As noted earlier, the TF1, TF2, and ETF soil samples were reddish brown, granular soils that contained no measurable organic component. The TNX soil was black, less crystalline, and contained organic debris. X-ray diffraction patterns for the 4060 mesh TF1, TP2, and TAX samples.revealed kaolinite and allicon oxide (sand). In different ratios for each soll. A small iraction of organic matter would not generate a crystalline pattern and could not be recognized in the spectra found in Appendix 1. The $X$-ray analysts of the BTF soll showed trace anounts of 1111te, kaolinite, and rutile clays in greater than 998 silicon oxide.

The reaultg of separation by slurrying gives a better picture of the ratios of sand to kaolinite in the TF1, TF2, and TrS samples. These are shown graphically in F1gure 1 for $40-60$ mesh and 80-100 mesh for those soils. Because of its residual redioactivity. apparently due to naturally occurring thorium and uranium, corresponding data was not generated for the ETP so11. 
D.L. FISH

WSRC-RP $-92-326$

March 20, 1992

Page 5 of 45

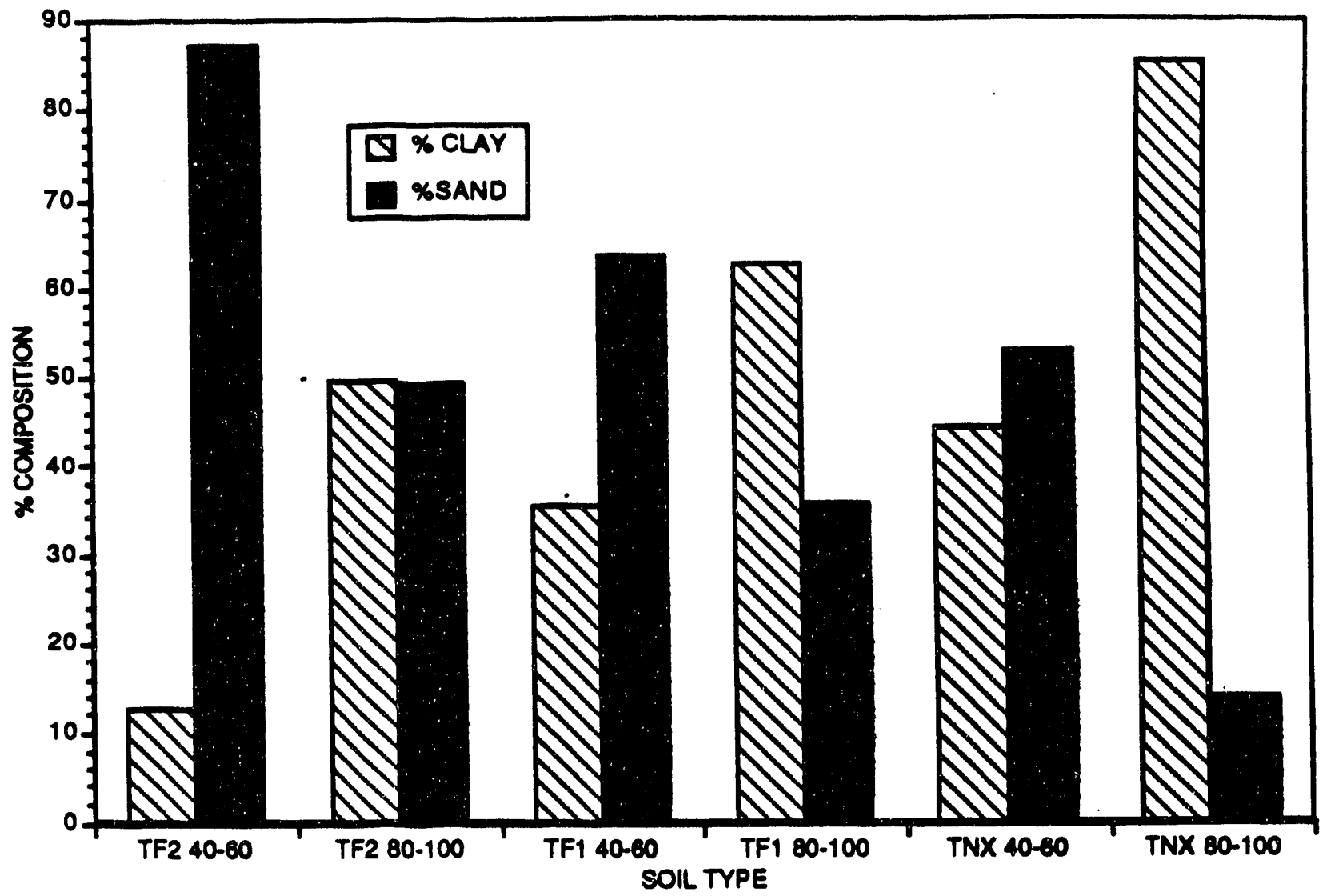

Figure 1. Sand and Kaolinite Percentages in Three SRs Soils of Mesh Sizes 40-60 and 80-100 
D.L. FISH

WSRC-RP-92-326

March 20, 1992

Page 6 of 45

Data for the determination of percent organic matter in the TNX soil are presented in Table 1.

Table 1. Percent Water and Organic Matter in TNX Soll (40-60 mesh) Soil weights, g

$\begin{array}{lccl}\text { conditions } & \text { Before } & \text { After } & \text { welght \& material } \\ 2 \text { hours at } 90^{\circ} \mathrm{C} & 5.00 & 4.94 & \begin{array}{l}1.28 \text { Interstitial } \\ \text { water }\end{array} \\ 2 \text { hours at } 150^{\circ} \mathrm{C} & 4.93 & 4.85 & 1.68 \text { bound water } \\ 16 \text { hours at } 600^{\circ} \mathrm{C} & 4.85 & 4.49 & 7.28 \text { organic matter }\end{array}$

\subsection{Bulk Donsities of Solis in soveral solutions}

Bulk densities of the solls in most of the solutions used for $\mathrm{Kd}$ determinations are recorded in Tables 2 and 3 . The purpose in using two tables for this data is to separate sieved (more uniformly sized, Table 3) soil data from unsleved soll (ETF) data, Table 2.

Table 2. Bulk Densities of ETF Soil

\begin{tabular}{|c|c|c|c|c|c|}
\hline Sample & Medium & مـ & g. Dry soil & mi wet soil & $\alpha_{\beta_{2}} \sigma / \mathrm{mL}_{1}$ \\
\hline $\begin{array}{l}\text { Deiol } \\
2 \\
3 \\
4 \\
5\end{array}$ & $\begin{array}{l}\text { hized water } \\
\text { Nitric acid } \\
\mathrm{NaOH} \\
\mathrm{KOH} \\
{ }_{1 \mathrm{M} \mathrm{CsNO}}\end{array}$ & $\begin{array}{r}4.45 \\
3.02 \\
10.01 \\
10.00 \\
6.60\end{array}$ & $\begin{array}{l}0.99 \\
1.00 \\
1.07 \\
1.03 \\
1.04\end{array}$ & $\begin{array}{l}0.90 \\
0.90 \\
0.80 \\
0.80 \\
0.80\end{array}$ & $\begin{array}{l}1.10 \\
1.11 \\
1.34 \\
1.29 \\
1.30\end{array}$ \\
\hline 6 & $0.01 \mathrm{M} \mathrm{vO} 2^{2+}$ & 3.00 & 1.03 & 1.00 & 1.03 \\
\hline 7 & $10 \mathrm{pm} \mathrm{Hg}^{2+}$ & 2.27 & 1.05 & 0.90 & 1.17 \\
\hline 3 & $2 \mathrm{M} \mathrm{Ca}\left(\mathrm{NO}_{3}\right)_{2}$ & 5.40 & 1.00 & 0.90 & 1.11 \\
\hline
\end{tabular}


Table 3. Bulk Densities of Three SRS Solls - 40-60 mesh

\begin{tabular}{|c|c|c|c|c|c|c|}
\hline $\begin{array}{r}\text { Sol } \\
\star \star \star \star \star \star \star\end{array}$ & 1 & 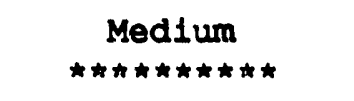 & $\underset{\star \mathrm{pH}}{\mathrm{pH}}$ & g Dry Sot1 & 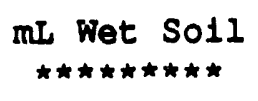 & 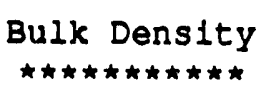 \\
\hline TF1 & 1 & Delonized Fater & 6.33 & 1.03 & 1.10 & 0.94 \\
\hline $\operatorname{TFI}$ & 2 & Nitric Acid & 1.22 & 1.01 & 1.10 & 0.92 \\
\hline TF1 & 3 & Nitric Acid & 3.02 & 1.02 & 1.20 & 0.85 \\
\hline TE1 & 4 & Nitric Actd & 5.05 & 1.01 & 1.20 & 0.84 \\
\hline TF1 & 5 & $\mathrm{NaOH}$ & 8.30 & 1.01 & 1.15 & 0.88 \\
\hline TF1 & 6 & $\mathrm{NaOH}$ & 10.01 & 1.03 & 1.00 & 1.03 \\
\hline TF1 & 7 & KOH & 10.00 & 1.00 & 1.10 & 0.91 \\
\hline TF1 & 8 & $10 \mathrm{ppm} \mathrm{Hg}$ (NO3) 2 & 2.27 & 1.03 & 1.80 & 0.57 \\
\hline TF1 & 9 & iM CsNO3 & 6.60 & 1.01 & 1.00 & 1.01 \\
\hline TE1 & 10 & $0.01 M 0022+$ & 3.00 & 1.00 & 1.10 & 0.91 \\
\hline TE1 & 11 & $2 \mathrm{M} \mathrm{Ca}(\mathrm{NO} 3)_{2}$ & 5.40 & 1.01 & 1.20 & 0.84 \\
\hline $\operatorname{TE} 1$ & 12 & 20 ppm Pb2+ & 5.63 & 1.01 & 1.00 & 1.01 \\
\hline TF2 & 1 & Delonized Water & 6.33 & 1.02 & 1.01 & 1.01 \\
\hline TF2 & 2 & Nitric Acld & 1.22 & 1.01 & 0.85 & 1.19 \\
\hline TE2 & 3 & Nitric Acid & 3.02 & 1.00 & 1.00 & 1.00 \\
\hline TE2 & 4 & Nitric Acid & 5.05 & 1.02 & 1.01 & 1.01 \\
\hline TF2 & 5 & $\mathrm{NaOH}$ & 8.30 & 1.02 & 0.90 & 1.13 \\
\hline TF2 & 6 & $\mathrm{NaOH}$ & 10.01 & 1.01 & 0.85 & 1.19 \\
\hline TE2 & 7 & KOH & 10.00 & 1.00 & 1.00 & 1.00 \\
\hline TF2 & 8 & $10 \mathrm{ppm} \mathrm{Hg}(\mathrm{NO} 3) 2$ & 2.27 & 1.03 & 1.20 & 0.86 \\
\hline TE2 & 9 & IM CsNO3 & 6.60 & 1.00 & 1.10 & 0.91 \\
\hline TF2 & 10 & $0.01 \mathrm{M} \mathrm{U02} \mathrm{2+}$ & 3.00 & 1.01 & 0.90 & 1.12 \\
\hline TF2 & 11 & $2 \mathrm{M} \mathrm{Ca}(\mathrm{NO} 3) 2$ & 5.40 & 1.03 & 1.00 & 1.03 \\
\hline TE2 & 12 & 20 ppm Pb2t & 5.63 & 1.00 & 0.80 & 1.25 \\
\hline TNX & 1 & Delonized Water & 6.19 & 1.02 & 1.70 & 0.60 \\
\hline TNX & 2 & N1tric Ac1d & 1.22 & 1.01 & 1.80 & 0.56 \\
\hline $\mathrm{TNX}$ & 3 & Nitric Acid & 3.02 & 1.01 & 1.80 & 0.56 \\
\hline TNX & 4 & Nitric Acid & 5.05 & 1.02 & 1.90 & 0.54 \\
\hline TNX & 5 & NaOH & 8.30 & 1.02 & 1.85 & 0.55 \\
\hline TNX & 6 & $\mathrm{NaOH}$ & 10.01 & 1.00 & 1.80 & 0.56 \\
\hline TNX & 7 & KOH & 10.00 & 1.03 & 1.70 & 0.61 \\
\hline TNX & 8 & $10 \mathrm{ppm} \mathrm{Hg}\left(\mathrm{NO}^{2}\right) 2$ & 2.27 & 1.03 & 0.90 & 1.14 \\
\hline TNX & 9 & IM CsNO3 & 6.60 & 1.00 & 1.50 & 0.67 \\
\hline TNX & 10 & $0.01 M$ U02 2t & 3.00 & 1.00 & 1.80 & 0.56 \\
\hline TNX & 11 & $2 \mathrm{MCa}(\mathrm{NO} 3)_{2}$ & 5.40 & 1.01 & 1.70 & 0.59 \\
\hline TNX & 12 & 20 ppm Pb2t & 5.63 & 1.00 & 1.40 & 0.72 \\
\hline
\end{tabular}




\subsection{DISTRIBUTION CONSTANTS-Rd}

\subsubsection{Soll $\mathrm{Kd}^{\prime}$ for $\mathrm{Bg}^{2+}$}

The Kd values derived for sorption of mercuric ion on two mesh sizes of TF1, TF2, and TNX solls and on ETF soll are presented in Tables 4-7. The notation for all duplicate samples in following tables is: The first sample in a set of duplicates will have the number $1,2,3, \ldots . n$ assoclated with its name. The duplicate sample will have the number designation $11,21,31, \ldots n 1$ associated with $1 t$. Duplicate samples differ slightly in the number of grams of soil used, but experienced the same initial solution in the experiments.

Table 4. Kd Values for $1-6 \mathrm{mg} / \mathrm{L}$ Mercuric Ion with TF1 Soil - 40-60 and 80-100 Mesh

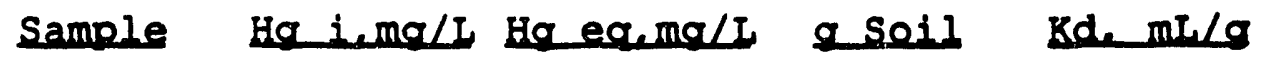

$\begin{array}{ccccc}\text { 40-60 Mesh } & & & & \\ \text { TF } 1-1 & 1.0800 & 0.0229 & 0.2029 & 3.41 \mathrm{E}+03 \\ \text { TF1-11 } & 1.0800 & 0.0103 & 0.2038 & 7.64 \mathrm{E}+03 \\ \text { TF } 1-2 & 2.3292 & 0.8600 & 0.2003 & 1.28 \mathrm{E}+02 \\ \text { TF1-21 } & 2.3292 & 0.8333 & 0.2092 & 1.29 \mathrm{E}+02 \\ \text { TF } 1-3 & 3.2919 & 2.2167 & 0.2173 & 3.35 \mathrm{E}+01 \\ \text { TF1-31 } & 3.2919 & 1.8500 & 0.2119 & 5.52 \mathrm{E}+01 \\ \text { TF } 1-4 & 6.6000 & 4.0645 & 0.2005 & 4.67 \mathrm{E}+01 \\ \text { TF } 1-41 & 6.6000 & 3.1290 & 0.2220 & 7.50 \mathrm{E}+01\end{array}$

80-100 Mesh

$\begin{array}{ccccc}\text { TF1-5 } & 1.0516 & 0.0112 & 0.2243 & 6.21 \mathrm{E}+03 \\ \text { TF1-51 } & 1.0516 & 0.0112 & 0.2159 & 6.45 \mathrm{E}+03 \\ \text { TE1-6 } & 2.3179 & 0.3944 & 0.2030 & 3.60 \mathrm{E}+02 \\ \text { TE1-61 } & 2.3179 & 0.3766 & 0.2020 & 3.83 \mathrm{E}+02 \\ \text { TE1-7 } & 3.0380 & 1.4873 & 0.2092 & 7.48 \mathrm{E}+01 \\ \text { TF1-71 } & 3.0380 & 1.5190 & 0.2269 & 6.61 \mathrm{E}+01 \\ \text { TE1-8 } & 5.8228 & 4.2722 & 0.2045 & 2.66 \mathrm{E}+01 \\ \text { TF1-81 } & 5.8228 & 4.0190 & 0.2048 & 3.29 \mathrm{E}+01\end{array}$

$i=$ Initial concentration

eq = Equilibrium concentration 
D.L. FISH

WSRC-RP-92-326

March 20, 1992

Page 9 of 45

Table 5. Kd Values for $1-6 \mathrm{mg} / \mathrm{L}$ Mercuric Ion With TE2 Soil - 40-60 and 80-100 Mesh

Sample $\mathrm{Hg} i \mathrm{mg} / \mathrm{L} \mathrm{Hg} \mathrm{eg} \mathrm{mg} / \mathrm{L}$ g soil $\mathrm{Kal} \mathrm{mL} / \mathrm{g}$

40-60 Mash

$\begin{array}{ccccc}\text { TF2-1 } & 1.0800 & 0.0994 & 0.2140 & 6.91 \mathrm{E}+02 \\ \text { TF2-11 } & 1.0800 & 0.0697 & 0.2141 & 1.02 \mathrm{E}+03 \\ \text { TF2-2 } & 2.3292 & 0.0413 & 0.2134 & 3.89 \mathrm{E}+03 \\ \text { TF2-21 } & 2.3292 & 0.9355 & 0.2155 & 1.04 \mathrm{E}+02 \\ \text { TF2-3 } & 3.2919 & 2.1613 & 0.2064 & 3.80 \mathrm{E}+01 \\ \text { TE2-31 } & 3.2919 & 2.0000 & 0.2161 & 4.48 \mathrm{E}+01 \\ \text { TF2-4 } & 6.6000 & 3.5161 & 0.2235 & 5.89 \mathrm{E}+01 \\ \text { TF2-41 } & 6.6000 & 4.4194 & 0.2195 & 3.37 \mathrm{E}+01\end{array}$

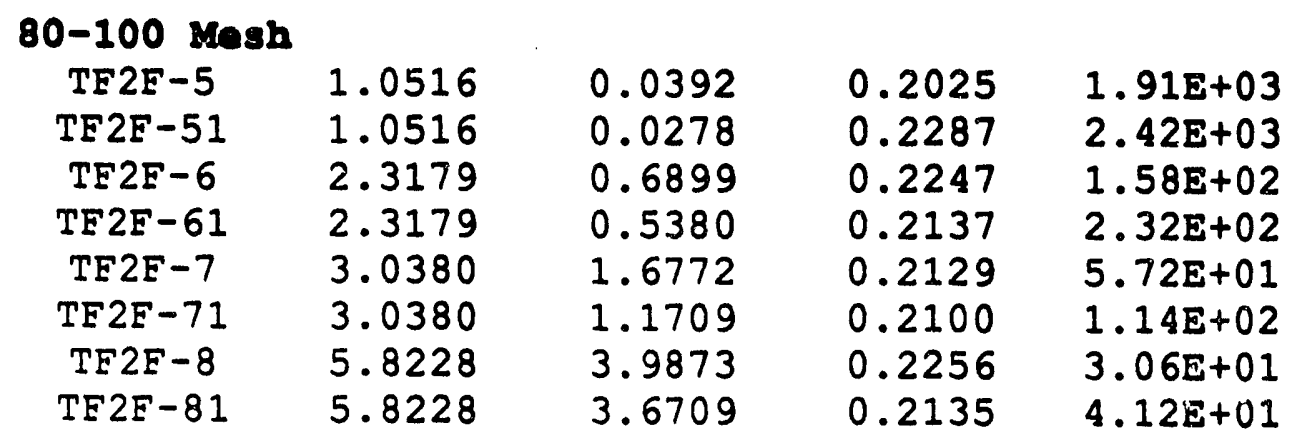

$i=$ Initial concentration

eq = Equilibrium concentration 
Table 6. Kd Values for $1-6 \mathrm{mg} / \mathrm{L}$ Mercuric Ion With TNX Soil - 40-60 and 80-100 Mesh

Sample $\mathrm{Hg} i \mathrm{mo} / \mathrm{L} \mathrm{Ha} \mathrm{eq.ma/L} \mathrm{a} \mathrm{soil} \mathrm{Ka} \mathrm{mL} / \mathrm{g}$

$\begin{array}{ccccc}\text { 40-60 Mash } & & & & \\ \text { TNX-1 } & 1.0800 & 0.0103 & 0.2028 & 7.68 \mathrm{E}+03 \\ \text { TNX-11 } & 1.0800 & 0.0103 & 0.2099 & 7.42 \mathrm{E}+03 \\ \text { TNX-2 } & 2.3292 & 0.0103 & 0.2027 & 1.67 \mathrm{E}+04 \\ \text { TNX-21 } & 2.3292 & 0.0103 & 0.2027 & 1.67 \mathrm{E}+04 \\ \text { TNX-3 } & 3.2919 & 0.0103 & 0.2024 & 2.36 \mathrm{E}+04 \\ \text { TNX-31 } & 3.2919 & 0.0103 & 0.2144 & 2.23 \mathrm{E}+04 \\ \text { TNX-4 } & 6.6000 & 0.1349 & 0.2086 & 3.45 \mathrm{E}+03 \\ \text { TNX-41 } & 6.6000 & 0.0960 & 0.2013 & 5.05 \mathrm{E}+03\end{array}$

\section{0-100 Mesh}

$\begin{array}{ccccc}\text { TNX-5 } & 1.0516 & 0.0119 & 0.2028 & 6.46 \mathrm{E}+03 \\ \text { TNX-51 } & 1.0516 & 0.0119 & 0.2136 & 6.14 \mathrm{E}+03 \\ \text { TNX-6 } & 2.3179 & 0.0119 & 0.2156 & 1.35 \mathrm{E}+04 \\ \text { TNX-61 } & 2.3179 & 0.0119 & 0.2231 & 1.30 \mathrm{E}+04 \\ \text { TNX-7 } & 3.0380 & 0.0119 & 0.2023 & 1.89 \mathrm{E}+04 \\ \text { TNX-71 } & 3.0380 & 0.0119 & 0.2155 & 1.77 \mathrm{E}+04 \\ \text { TNX-8 } & 5.8228 & 0.0758 & 0.2057 & 5.53 \mathrm{E}+03 \\ \text { TNX-81 } & 5.8228 & 0.0671 & 0.2028 & 6.34 \mathrm{E}+03\end{array}$

$i=$ Initial concentration eq = Equilibrium concentration

Table 7. Kd Values for $1-5 \mathrm{mg} / \mathrm{L}$ Mercuric Ion With ETE Soil

\begin{tabular}{|c|c|c|c|c|c|}
\hline Sample & $\mathrm{DH}$ & os soil & $\mathrm{Ha}$ i. $\mathrm{mg} / \mathrm{I}$ & $\mathrm{Ho}$ eq. $\mathrm{ma} / \mathrm{I}$ & $\mathrm{Kd} / \mathrm{mr} / \mathrm{g}$ \\
\hline $\begin{array}{l}\text { ETE-5 } \\
\text { ETE-3 } \\
\text { ET'F-2 } \\
\text { ETE-1 }\end{array}$ & $\begin{array}{l}3.030 \\
3.580 \\
3.760 \\
4.120\end{array}$ & $\begin{array}{l}0.1977 \\
0.2040 \\
0.1978 \\
0.2028\end{array}$ & $\begin{array}{l}5.0311 \\
3.5093 \\
2.1273 \\
1.0621\end{array}$ & $\begin{array}{l}2.3099 \\
1.8129 \\
0.5731 \\
0.0468\end{array}$ & $\begin{array}{r}8.94 E+01 \\
6.88 E+01 \\
2.06 E+02 \\
1.60 E+03\end{array}$ \\
\hline
\end{tabular}

$i=$ Initial concentration eq = Equilibrium concentration 
4.3.2 Mercury, $\mathrm{Hg}^{2+}, \mathrm{Kd}$. When $1 \mathrm{M}$ or $2 \mathrm{M} \mathrm{Ca}^{2+} 1 \mathrm{~s}$ Present

Solutions were tested in which mercuric nitrate and calcium nitrate were both present. Calcium ion was either $1 M$ or $2 M$ in concentration and was added to determine whether its presence would inhibit sorption of mercuric ion. The Rd values for these systems are in Tables 8-11.

Table 8. Kd Values for Mercuric Ion in the Presence of $1 M$ and $2 M$ Calcium Ion40-60 Mesh TE1 Soil

\section{$1 x \operatorname{Cn}(1903) 2$}

$\begin{array}{cllll}\text { TF1-HG-1M Ca-1 } & 1.0191 & 0.3759 & 0.2016 & 1.27 E+02 \\ \text { TE1-HG-1M Ca-11 } & 1.0191 & 0.3652 & 0.2026 & 1.33 E+02 \\ \text { TE1-HG-1M Ca-2 } & 1.9745 & 1.2910 & 0.2082 & 3.81 E+01 \\ \text { TF1-HG-1M Ca-21 } & 1.9745 & 1.2060 & 0.2022 & 4.73 E+01 \\ \text { TF1-HG-1M Ca-3 } & 3.0255 & 2.1280 & 0.2144 & 2.95 E+01 \\ \text { TF1-HG-1M Ca-31 } & 3.0255 & 2.2340 & 0.2170 & 2.45 E+01 \\ \text { TE1-HG-1M Ca-4 } & 5.7325 & 5.3900 & 0.2110 & 4.52 E+00 \\ \text { TE1-HG-1M Ca-41 } & 5.7325 & 4.1460 & 0.2258 & 2.54 E+01\end{array}$

\section{M Ca (NO3) 2}

$\begin{array}{cllll}\text { TE1-HG-2M Ca-1 } & 1.0191 & 0.4459 & 0.2277 & 8.47 \mathrm{E}+01 \\ \text { TF1-HG-2M Ca-11 } & 1.0191 & 0.5796 & 0.2140 & 5.32 \mathrm{E}+01 \\ \text { TE1-HG-2M Ca-2 } & 2.1338 & 1.4268 & 0.2081 & 3.57 \mathrm{E}+01 \\ \text { TF1-HG-2M Ca-21 } & 2.1338 & 1.4522 & 0.2063 & 3.41 \mathrm{E}+01 \\ \text { TF1-HG-2M Ca-3 } & 3.3758 & 2.4841 & 0.2044 & 2.63 E+01 \\ \text { TF1-HG-2M Ca-31 } & 3.3758 & 2.4841 & 0.2003 & 2.69 E+01 \\ \text { TE1-HG-2M Ca-4 } & 5.1911 & 4.7771 & 0.2040 & 6.37 E+00 \\ \text { TF1-HG-2M Ca-41 } & 5.1911 & 4.7134 & 0.2027 & 7.50 \mathrm{E}+00\end{array}$

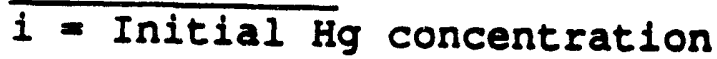
eq = Equilibrium $\mathrm{kg}$ concentration 
D.L. FISH

WSRC-RP-92-326

March 20, 1992

Page 12 of 45

Table 9. Kd Values for Mercuric Ion in the Presence of $1 \mathrm{M}$ and $2 \mathrm{M}$ Calcium Ion40-60 Mesh TE2 Soil

SAMPLE Hg $i, \mathrm{mg} / \mathrm{L} \mathrm{Hg}$ eq, $\mathrm{mg} / \mathrm{L}$ g Soil $\mathrm{Kd}, \mathrm{mL} / \mathrm{g}$

\begin{tabular}{|c|c|c|c|c|}
\hline $\begin{array}{l}1 \mathrm{Ca}(\mathrm{MO}) 2 \\
\text { TF2-HG-1M Ca-1 } \\
\text { TE2-HG-1M Ca-11 } \\
\text { TE2-HG-1M Ca-2 } \\
\text { TE2-HG-1M Ca-21 } \\
\text { TE2-HG-1M Ca-3 } \\
\text { TE2-HG-1M Ca-31 } \\
\text { TE2-HG-1M Ca-4 } \\
\text { TE2-HG-1M Ca-41 }\end{array}$ & $\begin{array}{l}1.0191 \\
1.0191 \\
1.9745 \\
1.9745 \\
3.0255 \\
3.0255 \\
5.7325 \\
5.7325\end{array}$ & $\begin{array}{l}0.3987 \\
0.4051 \\
1.4062 \\
1.4399 \\
2.3266 \\
2.4684 \\
5.0633 \\
5.1582\end{array}$ & $\begin{array}{l}0.2157 \\
0.2077 \\
0.2254 \\
0.2182 \\
0.2108 \\
0.2199 \\
0.2076 \\
0.2004\end{array}$ & $\begin{array}{l}1.08 E+02 \\
1.09 E+02 \\
2.69 E+01 \\
2.55 E+01 \\
2.14 E+01 \\
1.54 E+01 \\
9.55 E+00 \\
8.33 E+00\end{array}$ \\
\hline $\begin{array}{l}2 \mathrm{Ca}(\mathrm{NO} 3)^{2} \\
\text { TE2-HG-2M Ca-1 } \\
\text { TE2-HG-2M Ca-11 } \\
\text { TE2-HG-2M Ca-2 } \\
\text { TE2-HG-2M Ca-21 } \\
\text { TE2-HG-2M Ca-3 } \\
\text { TE2-HG-2M Ca-31 } \\
\text { TE2-HG-2M Ca-4 } \\
\text { TE2-HG-2M Ca-41 }\end{array}$ & $\begin{array}{l}1.0191 \\
1.0191 \\
2.1338 \\
2.1338 \\
3.3758 \\
3.3758 \\
5.1911 \\
5.1911\end{array}$ & $\begin{array}{l}0.7389 \\
0.7516 \\
1.6879 \\
1.7516 \\
2.7704 \\
2.3885 \\
4.6178 \\
4.7452\end{array}$ & $\begin{array}{l}0.2107 \\
0.2068 \\
0.2227 \\
0.2106 \\
0.2137 \\
0.2011 \\
0.2139 \\
0.2046\end{array}$ & $\begin{array}{l}2.70 E+01 \\
2.58 E+01 \\
1.78 E+01 \\
1.55 E+01 \\
1.53 E+01 \\
3.08 E+01 \\
8.71 E+00 \\
6.89 E+00\end{array}$ \\
\hline
\end{tabular}

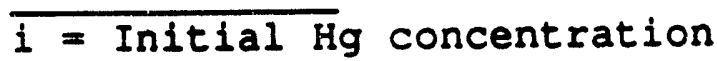
eq = Equilibrium $\mathrm{Hg}$ concentration 
Table 10. Kd Values for Mercuric Ion in the Presence of $1 \mathrm{M}$ and $2 \mathrm{M}$ Calcium Ion40-60 Mesh TNX Soil

SAMPLE $\mathrm{Hg} \mathrm{i,mg/L} \mathrm{Hg} \mathrm{eq,mg/L} \mathrm{g} \mathrm{soil} \mathrm{Kd}, \mathrm{mL} / \mathrm{g}$

$1 \mathrm{Ma}$ (NO3) 2

TNX-HG-1M Ca-1

1.0191

1.0191

TNX-HG-1M Ca-11

TNX-HG-IM Ca-2

TNX-HG-1M Ca-21

TNX-HG-1M Ca-3

TNX-HG-1M Ca-31

TNX-HG-1M Ca-4

TNX-HG-1M Ca-41

1.9745

1.9745

3.0255

3.0255

5.7325

5.7325

$2 \mathrm{Ca}$ (NO3) 2

\section{TNX-HG-2M Ca-1}

TNX-HG-2M-Ca-11

TNX-HG-2M-Ca-2

TNX-HG-2M-Ca-2I

TNX-HG-2M Ca-3

TNX-HG-2M Ca-31

TNX-HG-2M Ca-4

TNX-HG-2M Ca-41
1.0191

1.0191

2.1338

2.1338

3.3758

3.3758

5.1911

5.1911
0.0100

0.0100

0.0100

0.0100

0.0681

0.0780

0.4043

0.4184

0.0107

0.0107

0.0160

0.0102

0.1401

0.1783

0.7134

0.8662
$0.20337 .45 E+03$

$0.20087 .54 E+03$

$0.2117 \quad 1.39 \mathrm{E}+04$

$0.2179 \quad 1.35 E+04$

$0.21143 .08 E+03$

0.1998

$2.84 E+03$

0.2024

0.2034

9.77E+02

9. 37E+02

$i=$ Initial $\mathrm{Hg}$ concentration eq = Equilibrium $\mathrm{Hg}$ concentration

0.2030

0.2103

0.2039

0.2066

0.2113

0.2121

0.2087

0.2088
$6.96 \mathrm{E}+03$

$6.72 E+03$

$9.74 \mathrm{E}+03$

1. $51 \mathrm{E}+04$

1. $64 \mathrm{E}+03$

$1.27 \mathrm{E}+03$

4. $51 E+02$

$3.59 E+02$ 
Table 11. Kd Values for Mercuric Ion with ETE Soil in the Presence of $1 M$ and $2 M$ Calcium. Ion

SAMPLE Hg $i, \mathrm{mg} / \mathrm{L} \mathrm{Hg}$ eq, $\mathrm{mg} / \mathrm{L} \mathrm{g}$ Soil $\mathrm{Kd}, \mathrm{mL} / \mathrm{g}$

IM Ca (NO3) 2

ETE-HG-1M Ca-1

ETE-HG-1M Ca-11

ETE-HG-1M Ca-2

ETE-HG-1M Ca-21

ETE-HG-1M Ca-3

ETE-HG-1M Ca-31

ETE-HG-1M Ca-4

ETF-HG-1M Ca-41
3.8562

3.8562

4.2810

4.2810

1.0327

1.0327

0.9150

0.9150
4.2466

4.4863

4.6667

5.1852

0.1747

0.1918

0.3259

0.2333
0.2243

0.2165

0.2174

0.2170

0.2123

0.2161

0.2075

0.2023
$-6.15 E+00$

$-9.73 E+00$

$-5.70 E+00$

$-1.21 E+01$

$3.47 E+02$

$3.04 \mathrm{E}+02$

1. $31 E+02$

2.17E+02

\section{M Ca (NO3) 2}

ETE-HG-2M Ca-1
ETE-HG-2M-Ca-11
ETE-HG-2M-Ca-2
ETE-HG-2M-Ca-21
ETE-HG-2M Ca-3
ETE-HG-2M Ca-31
ETE-HG-2M Ca-4
ETE-HG-2M Ca-41

1.7974

1.4247

0.2012

1. $95 \mathrm{E}+01$

1.7974

1.3288

2.1569

1.4658

2.1569

1.0621

1.0621

2.7451

2.7451

1.3973

2. 3151

0.2061

2.57E+01

0.2025

3. $49 \mathrm{E}+01$

0.2065

$3.95 E+01$

0.8296

0.2109

$-3.85 E+01$

2.5856

0.2041

$2.06 \mathrm{E}+01$

2.3116

0.2109

$4.39 \mathrm{E}+00$

0.2033

$1.38 E+01$

$i=$ Initial Hg concentration

eq = Equilibrium Hg concentration 
D.I. FISB

WSRC-RP-92-326

March 20, 1992

Page 15 of 45

\subsubsection{Mercury, Bg ${ }^{2+}$, Kd's With Added Bt Present}

Solutions containing about $5 \mathrm{mg} / \mathrm{L} \mathrm{Hg}^{2+}$ and varying amounts of acid were contacted with the four soils to determine the effect of hydrogen ion on the ability of the soils to sorb mercuric ion. The Rd's resulting from these tests are found in Tables 12-15.

Table 12. Hg Kd Values for $5 \mathrm{mg} / \mathrm{L}$ Mercuric Ion and Different $\mathrm{pH}$ with TF1 Soil - 40-60 Mesh

Sample Hg $i, m g / L$ [H] i Hg eq,mg/L [H] eq $g$ soil Hg $\mathrm{Kd}$

\begin{tabular}{ccccccccc}
\hline TF1-PH1 & 5.20 & $7.08 \mathrm{E}-02$ & & 4.35 & $6.76 \mathrm{E}-02$ & 0.2205 & $1.33 \mathrm{E}+01$ \\
TF1-PH11 & 5.20 & & $7.08 \mathrm{E}-02$ & & 5.00 & $6.76 \mathrm{E}-02$ & 0.2061 & $2.91 \mathrm{E}+00$ \\
TF1-PH2 & 5.86 & $1.05 \mathrm{E}-02$ & & 4.09 & & $9.77 \mathrm{E}-03$ & 0.2100 & $3.10 \mathrm{E}+01$ \\
TF1-PH21 & 5.86 & $1.05 \mathrm{E}-02$ & 4.28 & $9.77 \mathrm{E}-03$ & 0.2077 & $2.66 \mathrm{E}+01$ \\
TF1-PH3 & 5.53 & $3.39 \mathrm{E}-03$ & 4.02 & $2.63 \mathrm{E}-03$ & 0.2106 & $2.68 \mathrm{E}+01$ \\
TF1-PH31 & 5.53 & $3.39 \mathrm{E}-03$ & 4.44 & $2.45 \mathrm{E}-03$ & 0.2118 & $1.73 \mathrm{E}+01$ \\
TF1-PH4 & 5.59 & $3.24 \mathrm{E}-03$ & 3.99 & $2.29 \mathrm{E}-03$ & 0.2100 & $2.87 \mathrm{E}+01$ \\
TF1-PH41 & 5.59 & $3.24 \mathrm{E}-03$ & 4.15 & $2.40 \mathrm{E}-03$ & 0.2043 & $2.55 \mathrm{E}+01$
\end{tabular}

$i=$ initial

eq = equilibrium

Table 13. Hg Rd Values for $5 \mathrm{mg} / \mathrm{L}$ Mercuric Ion and Different $\mathrm{pH}$ With TF2 Soil - 40-60 Mesh

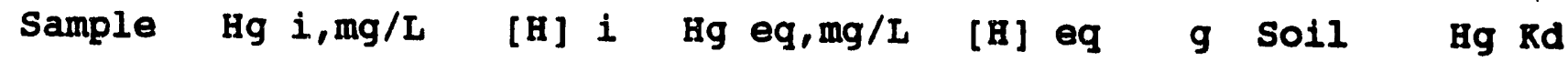

\begin{tabular}{|c|c|c|c|c|c|c|}
\hline TF1-PH1 & 5.20 & $7.08 \mathrm{E}-02$ & 4.35 & $6.76 \mathrm{~B}-02$ & 0.2205 & $1.33 \mathrm{~B}+01$ \\
\hline TF1-PH11 & 5.20 & $7.08 \mathrm{E}-02$ & 5.00 & $6.76 \mathrm{~B}-02$ & 0.2061 & $2.91 \mathrm{~B}+00$ \\
\hline TF1-PH2 & 5.86 & $1.05 \mathrm{E}-02$ & 4.09 & $9.77 \mathrm{E}-03$ & 0.2100 & $3.10 \mathrm{E}+01$ \\
\hline TF1-PE21 & 5.86 & $1.05 \mathrm{E}-02$ & 4.28 & $9.77 \mathrm{E}-03$ & 0.2077 & $2.66 \mathrm{~B}+01$ \\
\hline TF1-PH3 & 5.53 & $3.39 \mathrm{E}-03$ & 4.02 & $2.63 \mathrm{E}-03$ & 0.2106 & $2.68 \mathrm{~B}+01$ \\
\hline TF1-PB31 & 5.53 & $3.39 \mathrm{E}-03$ & 4.44 & $2.45 \mathrm{E}-03$ & 0.2118 & $1.73 E+01$ \\
\hline TF1-PB4 & 5.59 & $3.24 \mathrm{E}-03$ & 3.99 & $2.29 \mathrm{E}-03$ & 0.2100 & $2.87 B+01$ \\
\hline TE1-PH41 & 5.59 & $3.24 \mathrm{E}-03$ & 4.15 & $2.40 \mathrm{E}-03$ & 0.2043 & $2.55 B+01$ \\
\hline
\end{tabular}

$i=$ initial

eq a equilibrium 
Table 14. Hg Kd Values for $5 \mathrm{mg} / \mathrm{L}$ Mercuric Ion and Different $\mathrm{pH}$ with TNX Soil - 40-60 Mesh

\begin{tabular}{|c|c|c|c|c|c|c|}
\hline Sample & $\mathrm{Bg} i, \mathrm{ppm}$ & [B] $i$ & $\mathrm{Hg}$ eq,ppm & [H] eq & Soil & Hg Rd \\
\hline TNX-PBI & 5.20 & $7.08 \mathrm{~g}-02$ & 1.17 & $6.92 \mathrm{E}-02$ & 0.2040 & $2.53 \mathrm{~B}+02$ \\
\hline TNX-PB11 & 5.20 & $7.08 \mathrm{~B}-02$ & 1.33 & $6.61 \mathrm{E}-02$ & 0.2138 & $2.04 \mathrm{~B}+02$ \\
\hline TWX-PB2 & 5.86 & $1.05 \mathrm{E}-02$ & 1.17 & $8.91 \mathrm{E}-03$ & 0.2077 & $2.89 \mathrm{~B}+02$ \\
\hline TNX-PB21 & 5.86 & $1.05 B-02$ & 1.60 & $9.12 \mathrm{E}-03$ & 0.2000 & $2.00 \mathrm{~B}+02$ \\
\hline TWX-PB3 & 5.53 & $3.39 \mathrm{~B}-03$ & 0.60 & $2.45 \mathrm{~B}-03$ & 0.2003 & $6.16 \mathrm{~B}+02$ \\
\hline TNX-PB31 & 5.53 & $3.39 \mathrm{E}-03$ & 0.47 & $2.63 \mathrm{z}-03$ & 0.2123 & $7.54 \mathrm{E}+02$ \\
\hline TWX $=$ PB4 & 5.59 & $3.24 B-03$ & 0.76 & $2.45 \mathrm{~B}-03$ & 0.2014 & $4.76 \mathrm{~B}+02$ \\
\hline TNX-PB41 & 5.59 & $3.24 \mathrm{E}-03$ & 0.54 & $2.45 \mathrm{E}-03$ & 0.2013 & $6.97 \mathrm{~B}+02$ \\
\hline
\end{tabular}

$i=$ initial

eq = equilibriun

Table 15. Hg Rd Values for $5 \mathrm{mg} / \mathrm{L}$ Mercuric Ion and Different $\mathrm{pH}$ with ETF Soil

Sample Hg $i, m g / L$ [H] $i$ Hg eq, $\mathrm{ng} / \mathrm{L}$ [B] eq $g$ Soil $\mathrm{Bg}$ Rd

\begin{tabular}{|c|c|c|c|c|c|c|}
\hline ETF-HgB-1 & 4.941 & $7.24 \mathrm{~B}-02$ & 3.856 & $6.08 \mathrm{E}-02$ & 0.2654 & $1.59 \mathrm{E}+01$ \\
\hline ETF-HgH-11 & 4.941 & $7.24 \mathrm{E}-02$ & 4.379 & $6.07 \mathrm{E}-02$ & 0.2324 & $8.28 \mathrm{~B}+00$ \\
\hline ETF-BgB-2 & 4.941 & $7.59 \mathrm{E}-03$ & 4.381 & $6.21 \mathrm{E}-03$ & 0.2351 & $8.16 \mathrm{~B}+00$ \\
\hline ETF-BgE-21 & 4.941 & $7.59 \mathrm{E}-03$ & 4.673 & $6.43 \mathrm{E}-03$ & 0.2439 & $3.53 \mathrm{~B}+00$ \\
\hline ETF-BgB-3 & 4.762 & $8.32 \mathrm{E}-04$ & 2.843 & $4.24 \mathrm{E}-04$ & 0.2213 & $4.58 \mathrm{~B}+01$ \\
\hline ETF-BgB-31 & 4.762 & $8.32 \mathrm{~B}-04$ & 1.895 & $5.63 \mathrm{E}-04$ & 0.2464 & $9.21 \mathrm{~B}+01$ \\
\hline ETF-BgH-4 & 5.06 & $3.63 \mathrm{~B}-03$ & 3.758 & $1.45 \mathrm{E}-03$ & 0.2663 & $1.95 \mathrm{~B}+01$ \\
\hline ETF-BgE-41 & 5.06 & $3.63 \mathrm{~B}-03$ & 4.248 & $1.45 \mathrm{E}-03$ & 0.2470 & $1.16 \mathrm{~B}+01$ \\
\hline
\end{tabular}

$i$ = initial

eq = equilibrium 
D.I. FISH

WSRC-RP-92-326

March 20, 1992

Page 17 of 45

\subsubsection{Soll Kd's for Iead, $\mathrm{Pb}^{2+}$}

The $\mathrm{Kd}$ values derived for sorption of lead ion on 40-60 mesh samples of TF1, TF2, and TNX soils and on ETF soil are presented in Tables 16-19.

Table 16. Kd Values for 50-125 $\mathrm{mg} / \mathrm{I}$ Lead Ion With Tr1 Soil - 40-60 Mesh

Sample $\mathrm{Pb} i, m g / L \mathrm{~Pb}$ eq, $\mathrm{mg} / \mathrm{I} g$ soil $\mathrm{Kd}, \mathrm{mL} / \mathrm{g}$

\begin{tabular}{ccccc}
\hline & & & & \\
\cline { 2 - 6 } TF1-P1 & 47.428 & $<0.020$ & 0.5145 & $\geq 6.91 \mathrm{~B}+04$ \\
TE1-P11 & 47.428 & $<0.020$ & 0.5138 & $\geq 6.92 \mathrm{~B}+04$ \\
TF1-P2 & 71.654 & $<0.020$ & 0.5127 & $\geq 1.05 \mathrm{~B}+05$ \\
TF1-P21 & 71.654 & $<0.020$ & 0.4997 & $\geq 1.08 \mathrm{~B}+05$ \\
TF1-P3 & 97.935 & $<0.020$ & 0.5089 & $\geq 1.44 \mathrm{~B}+05$ \\
TF1-P31 & 97.935 & $<0.020$ & 0.5025 & $\geq 1.46 \mathrm{~B}+05$ \\
TF1-P4 & 121.400 & 0.241 & 0.5051 & $1.49 \mathrm{~B}+04$ \\
TE1-P41 & 121.400 & 0.466 & 0.5000 & $7.79 \mathrm{~B}+03$
\end{tabular}

$i=$ initial

eq = equilibrium

Table 17. Rd Values for 50-125 mg/L Lead Ion With TF2 Soil - 40-60 Mesh

Sample $\mathrm{Pb} i, \mathrm{mg} / \mathrm{L} \mathrm{Pb}$ eq,mg/L $g$ soil $\mathrm{Kd}, \mathrm{mr} / \mathrm{g}$

\begin{tabular}{|c|c|c|c|c|}
\hline TR2-P1 & 47.428 & 2.772 & 0.5010 & $4.82 \mathrm{~B}+02$ \\
\hline TF2-P11 & 47.428 & 1.459 & 0.5110 & $9.25 \mathrm{~B}+02$ \\
\hline TP2-P2 & 71.654 & 10.982 & 0.5089 & $1.63 \mathrm{~B}+02$ \\
\hline TF2-P21 & 71.654 & 13.372 & 0.5026 & $1.30 z+02$ \\
\hline TF2-P3 & 97.935 & 24.212 & 0.5100 & $8.96 \mathrm{~B}+01$ \\
\hline TF2-P31 & 97.935 & 17.797 & 0.5135 & $1.32 \mathrm{~B}+02$ \\
\hline TE2-P4 & 121.400 & 37.444 & 0.5143 & $6.54 \mathrm{~B}+01$ \\
\hline$T E 2-P 41$ & 121.400 & 38.651 & 0.5125 & $6.27 \mathrm{~B}+01$ \\
\hline
\end{tabular}

$\overline{i=i n i t i a l}$

eq = equilibrium 
Table 18. Rd Values for 50-125 $\mathrm{mg} / \mathrm{L}$ Lead Ion with TNX Soil - 40-60 Mesh

Sample $\mathrm{Pb} i, \mathrm{mg} / \mathrm{L} \mathrm{Pb}$ eq,mg/L g Soil $\mathrm{Rd}, \mathrm{mL} / \mathrm{g}$

\begin{tabular}{cccccc}
\hline & & & & & \\
TWX-P1 & 47.428 & $<0.02$ & 0.5010 & $>7.03 \mathrm{~B}+03$ \\
TWX-P11 & 47.428 & $<0.02$ & 0.5110 & $>6.95 \mathrm{~B}+03$ \\
TWX-P2 & 71.654 & 0.037 & 0.5089 & $5.718+04$ \\
TWX-P21 & 71.654 & 0.028 & 0.5026 & $7.63 \mathrm{~B}+04$ \\
TWX-P3 & 97.935 & $<0.02$ & 0.5100 & $>1.42 \mathrm{~B}+05$ \\
TWX-P31 & 97.935 & $<0.02$ & 0.5135 & $>1.46 \mathrm{~B}+05$ \\
TWX-P4 & 121.400 & 0.025 & 0.5143 & $1.42 \mathrm{~B}+05$ \\
TWX-P41 & 121.400 & $<0.02$ & 0.5125 & $>1.77 \mathrm{~B}+05$
\end{tabular}

$i=$ initial

eq = equilibrium

Table 19. Kd Values for $5-50 \mathrm{mg} / \mathrm{L}$ Lead Ion with ETF Soi]

Sample $\mathrm{Pb} i, \mathrm{mg} / \mathrm{L} \mathrm{Pb}$ eq, mg./L g soil $\mathrm{Rd}, \mathrm{mu} / \mathrm{g}$

$\begin{array}{lcccc}\text { ETF-1 } & 35.370 & 0.203 & 0.1020 & 2.42 \mathrm{E}+06 \\ \text { ETF-11 } & 35.370 & 0.823 & 0.1066 & 5.61 \mathrm{~B}+05 \\ \text { ETF-2 } & 12.276 & <0.02 & 0.1077 & \geq 8.11 \mathrm{E}+06 \\ \text { ETF-21 } & 12.276 & <0.02 & 0.1014 & \geq 8.61 \mathrm{~B}+06 \\ \text { ETF-3 } & 6.916 & <0.02 & 0.1056 & \geq 4.65 \mathrm{~B}+06 \\ \text { ETF-31 } & 6.916 & 0.027 & 0.1067 & 3.41 \mathrm{~B}+06 \\ \text { ETF-4 } & 3.150 & <0.02 & 0.1031 & \geq 2.16 \mathrm{~B}+06 \\ \text { ETF-41 } & 3.150 & <0.02 & 0.1028 & \geq 2.17 \mathrm{E}+06\end{array}$

. $i=$ Initial $\mathrm{Pb}$ concentration

Q = Bquilibriun Pb Concentration 
D.I. FISE

WSRC-RP-92-326

March 20, 1992

Page 19 of 45

4.3.5 Lead, $\mathrm{Pb}^{2+}, \mathrm{Kd}$ 's When $1 \mathrm{M}$ or $2 \mathrm{M} \mathrm{Ca}^{2+}$ is Present

The Kd values observed for sorption of lead ion from solutions of lead nitrate in the presence of $1 M$ or $2 M$ calcium nitrate are presented in Tables 20-23.

Table 20. Kd Values for Lead Ion in the Presence of $1 M$ and $2 M$ Calcium Ion

40-60 Mesh TF1 Soil

SAMPLE $\mathrm{Pb}$ i, $\mathrm{mg} / \mathrm{L} \mathrm{Pb}$ eq, $\mathrm{mg} / \mathrm{L} \mathrm{g}$ Soil $\mathrm{Kd}, \mathrm{mg} / \mathrm{L}$

1M Ca ( $\mathrm{NO3}^{2} 2$

$\begin{array}{ccccc}\text { TE } 1-5 & 42.600 & 14.100 & 0.5093 & 5.96 E+01 \\ \text { TEI-51 } & 42.600 & 20.300 & 0.5070 & 3.26 E+01 \\ \text { TE } 1-6 & 66.800 & 31.400 & 0.5159 & 3.28 E+01 \\ \text { TE1-61 } & 66.800 & 31.100 & 0.5124 & 3.36 \mathrm{E}+01 \\ \text { TE } 1-7 & 86.288 & 48.041 & 0.5042 & 2.37 E+01 \\ \text { TE } 1-71 & 86.288 & 54.417 & 0.5127 & 1.71 E+01 \\ \text { TE } 1-8 & 153.810 & 114.330 & 0.5139 & 1.01 E+01 \\ \text { TE1-81 } & 153.810 & 105.240 & 0.5098 & 1.36 \mathrm{E}+01\end{array}$

\section{$2 \mathrm{M} \mathrm{Ca}(\mathrm{NO}) 2$}

$\begin{array}{cllll}\text { TF1CA-1 } & 35.488 & 12.269 & 0.5061 & 5.61 \mathrm{E}+01 \\ \text { TF1CA-11 } & 35.488 & 14.944 & 0.4999 & 4.13 \mathrm{E}+01 \\ \text { TE1CA-2 } & 55.976 & 26.229 & 0.5197 & 3.27 \mathrm{E}+01 \\ \text { TE1CA-21 } & 55.976 & 30.426 & 0.5101 & 2.47 \mathrm{E}+01 \\ \text { TF1CA-3 } & 77.028 & 43.999 & 0.5037 & 2.24 \mathrm{E}+01 \\ \text { TE1CA-31 } & 77.028 & 45.530 & 0.5002 & 2.07 \mathrm{E}+01 \\ \text { TE1CA-4 } & 96.556 & 60.878 & 0.5121 & 1.72 \mathrm{E}+01 \\ \text { TE1CA-41 } & 96.556 & 56.138 & 0.5256 & 2.05 \mathrm{E}+01\end{array}$

$i=$ initial

eq = equilibrium 
Table 21. Kd Values for Lead Ion in the Presence of $1 \mathrm{M}$ and $2 \mathrm{M}$ Calcium Ion - 40-60 Mesh TF2 Soil

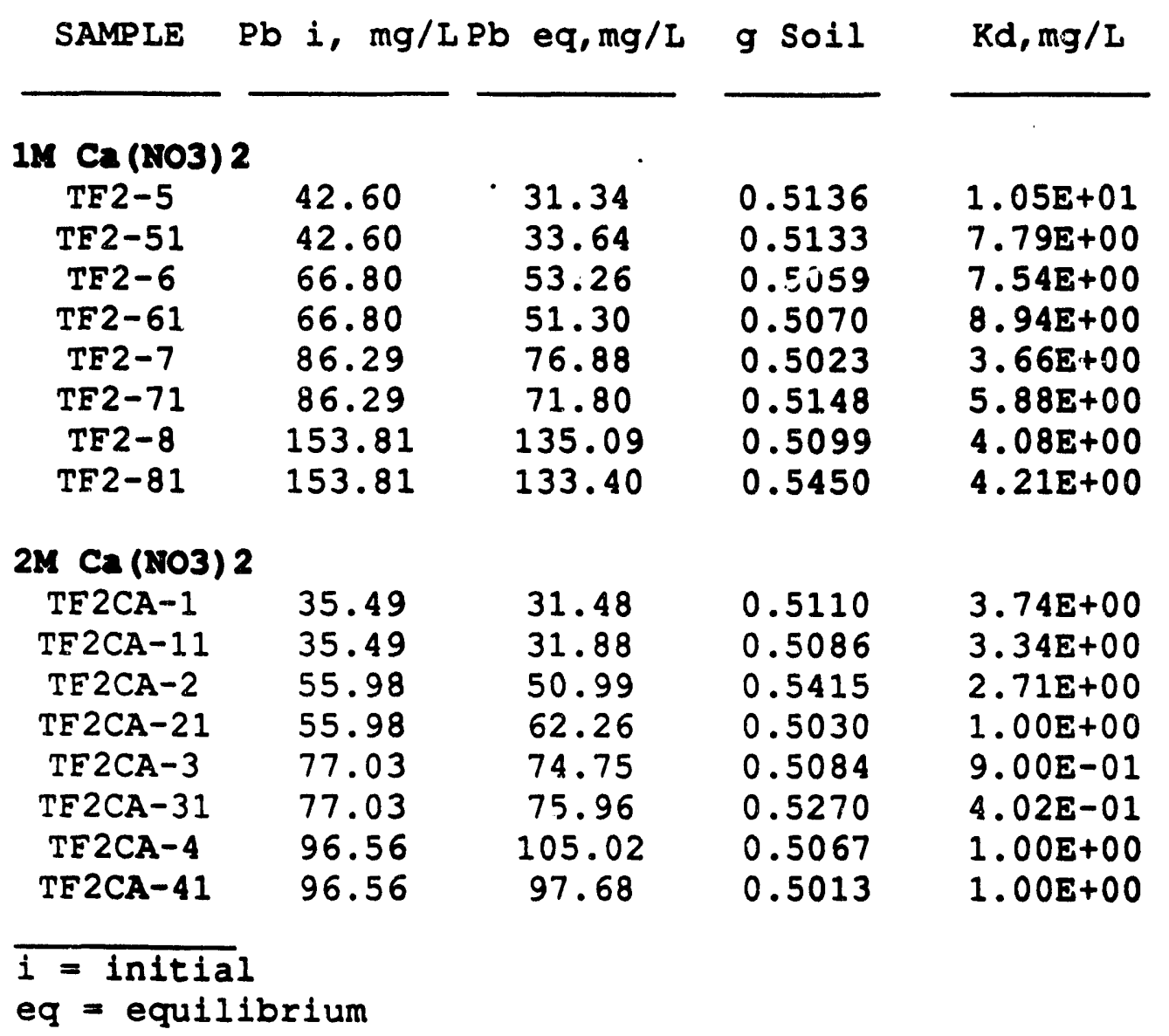


Table 22. Kd Values for Lead Ion in the Presence of $1 \mathrm{M}$ and 2M Calcium Ion - 40-60 Mesh TNX Soil

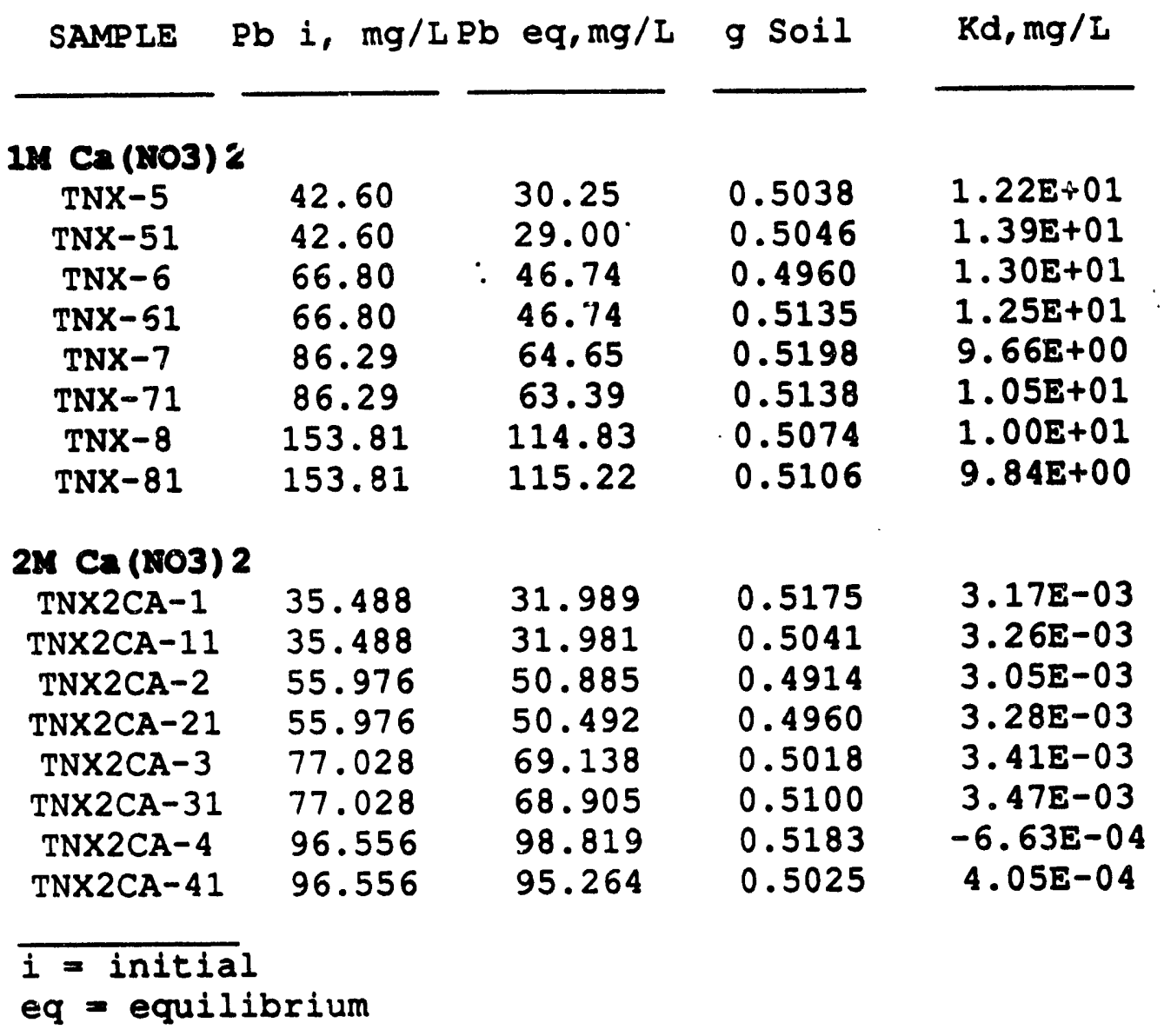


Table 23. Kd Values for Lead Ion in the Presence of $1 M$ and $2 M$ Calcium Ion - ETF Soil

SAMPLE $\mathrm{Pb} i, \mathrm{mg} / \mathrm{LPb}$ eq, $\mathrm{mg} / \mathrm{L} \mathrm{g}$ Soil $\mathrm{Kd}, \mathrm{mg} / \mathrm{L}$

14. Ca (NO3) 2

$\begin{array}{ccccc}\text { ETF-5 } & 42.60 & 20.36 & 0.5040 & 3.25 E+01 \\ \text { ETE-51 } & 42.60 & 18.77 & 0.5057 & 3.77 E+01 \\ \text { ETF-6 } & 66.80 & 29.92 & 0.5082 & 3.64 E+01 \\ \text { ETE-61 } & 66.80 & 34.23 & 0.4953 & 2.88 E+01 \\ \text { ETF-7 } & 86.29 & 52.28 & 0.5038 & 1.94 E+01 \\ \text { ETF-71 } & 86.29 & 52.67 & 0.5066 & 1.89 E+01 \\ \text { ETF-8 } & 153.81 & 120.14 & 0.5080 & 8.28 E+00 \\ \text { ETE-81 } & 153.81 & 118.33 & 0.5077 & \mathbf{8 . 8 E E + 0 0}\end{array}$

2x Ca (No3) 2

$\begin{array}{ccccc}\text { ETF2CA-1 } & 41.96 & 24.14 & 0.5069 & 2.19 E+01 \\ \text { ETF2CA-11 } & 41.96 & 27.86 & 0.5014 & 1.51 \mathrm{E}+01 \\ \text { ETF2CA-2 } & 57.95 & 38.50 & 0.5070 & 1.49 E+01 \\ \text { ETF2CA-21 } & 57.95 & 45.29 & 0.5094 & 8.23 \mathrm{E}+00 \\ \text { ETF2CA-3 } & 89.61 & 56.58 & 0.5084 & 1.72 \mathrm{E}+01 \\ \text { ETF2CA-31 } & 89.61 & 65.62 & 0.4997 & 1.10 \mathrm{E}+01 \\ \text { ETE2CA-4 } & 144.99 & 116.30 & 0.5067 & 7.30 \mathrm{E}+00 \\ \text { ETF2CA-41 } & 144.99 & 123.48 & 0.5056 & 5.17 \mathrm{E}+00\end{array}$

$i=$ Initial

eq = equilibrium 
D.I. EISH

WSRC-RP-92-326

March 20, 1992

Page 23 of 45

\subsubsection{Laad, $\mathrm{Pb}^{2+}, \mathrm{Kd}=$ with Added $\mathrm{g}^{+}$present}

Solutions containing about $100 \mathrm{mg} / \mathrm{L} \mathrm{Pb}^{2+}$ and varying amounts of acid were contacted with the four soils to determine the effect of hydrogen ion on the ability of the solls to sorb lead ion. The Kd's resulting from these tests are found in Tables 24-27.

Table 24. $\mathrm{Pb} \mathrm{Kd}$ Values for $100 \mathrm{mg} / \mathrm{L}$ Lead Ion and Different $\mathrm{pH}$ With TEl Soil - 40-60 Mesh

Sample $\mathrm{Pb} i, \mathrm{mg} / \mathrm{L}[\mathrm{H}] \mathrm{i} \mathrm{Pb}$ eq, $\mathrm{mg} / \mathrm{L}[\mathrm{H}]$ eq $g$ Soil $\mathrm{Pb} \mathrm{Kd}$

$\begin{array}{cc}\text { TF1PH-1 } & 97.2 \\ \text { TF1PH-11 } & 97.2 \\ \text { TF1PH-2 } & 105.06 \\ \text { TF1PH-21 } & 105.06 \\ \text { TF1PH-3 } & 108.67 \\ \text { TF1PH-31 } & 108.67 \\ \text { TF1PH-4 } & 104.70 \\ \text { TF1PH-41 } & 104.70 \\ & \\ & \\ \text { e initial } & \\ \text { e equilibrium }\end{array}$

Table 25. $\mathrm{Pb} \mathrm{Kd}$ Values for $100 \mathrm{mg} / \mathrm{L}$ Lead Ion at Different $\mathrm{pH}$ Values with TE2 Soil - 40-60 Mesh

Sample $\mathrm{Pb} i, \mathrm{mg} / \mathrm{L}[\mathrm{H}]$ i $\mathrm{Pb}$ eq, $\mathrm{mg} / \mathrm{L}$ [H] eq $g$ soil $\mathrm{Pb} \mathrm{Kd}$

\begin{tabular}{|c|c|c|c|c|c|c|}
\hline $\begin{array}{l}\text { TE2PH-1 } \\
\text { TE2PH-11 } \\
\text { TE2PH-2 } \\
\text { TF } 2 \text { PH-21 } \\
\text { TE2PH-3 } \\
\text { TE2PH-31 } \\
\text { TE2PH-4 } \\
\text { TF2PH-41 }\end{array}$ & $\begin{array}{c}97.21 \\
97.21 \\
105.06 \\
105.06 \\
108.67 \\
108.67 \\
104.70 \\
104.70\end{array}$ & $\begin{array}{l}3.50 E-01 \\
3.50 E-01 \\
3.09 E-02 \\
3.09 E-02 \\
1.66 E-03 \\
1.66 E-03 \\
2.45 E-05 \\
2.45 E-05\end{array}$ & $\begin{array}{c}93.67 \\
93.98 \\
100.40 \\
100.38 \\
93.34 \\
93.52 \\
36.04 \\
32.74\end{array}$ & $\begin{array}{l}5.50 E-01 \\
6.03 E-01 \\
8.13 E-02 \\
2.88 E-02 \\
8.51 E-04 \\
8.32 E-04 \\
4.27 E-06 \\
3.02 E-06\end{array}$ & $\begin{array}{l}0.5163 \\
0.5335 \\
0.5123 \\
0.5163 \\
0.5139 \\
0.5009 \\
0.5098 \\
0.5316\end{array}$ & $\begin{array}{l}4.43 E+01 \\
4.42 E+01 \\
5.08 E+02 \\
5.08 E+02 \\
1.05 E+04 \\
1.05 E+04 \\
1.78 E+06 \\
1.96 E+06\end{array}$ \\
\hline
\end{tabular}

$i=$ initial

eq $=$ equilibrium 
D.L. FISH

WSRC-RP $-92-326$

March 20, 1992

Page 24 of 45

Table 26. $\mathrm{Pb} \mathrm{Kd}$ Values for $100 \mathrm{mg} / \mathrm{L}$ Lead Ion at Different $\mathrm{pH}$ Values With TNX Soil - 40-60 Mesh

Sample $\mathrm{Pb} i, \mathrm{mg} / \mathrm{L}$ [H] i $\mathrm{Pb}$ eq, $\mathrm{mg} / \mathrm{L}$ [H] eq g soil $\mathrm{Pb} \mathrm{Kd}$

\begin{tabular}{|c|c|c|c|c|c|c|}
\hline TNXPH-1 & & 6 & & 1 & 05090 & \\
\hline TNXPH-11 & 97.21 & 3. $50 \mathrm{E}-01$ & 101.87 & $5.23 \mathrm{E}-01$ & 0.5184 & $\begin{array}{l}-2.37 E+00 \\
-1.96 E+00\end{array}$ \\
\hline TNXPH-2 & 105.06 & $.09 E-02$ & 102.54 & $4.16 \mathrm{E}-02$ & 0.5013 & $1.19 \mathrm{E}+01$ \\
\hline TNXPH -21 & & $3.09 E-02$ & 102.82 & $4.68 \mathrm{E}-02$ & 042 & $1.06 \mathrm{E}+01$ \\
\hline TNXPH-3 & & 1.66 & 67 & 04 & 0 & $1.40 E+04$ \\
\hline TNXPH-31 & 10 & $1.66 \mathrm{E}-03$ & 36.17 & 04 & 0.5232 & $1.81 E+04$ \\
\hline TNXPH-4 & .70 & $2.45 E-05$ & 0.11 & 1.58 & 0.5106 & $5.67 \mathrm{E}+08$ \\
\hline TNXPH-41 & 104.70 & $2,45 \mathrm{E}-05$ & 0.15 & $1.86 \mathrm{E}-07$ & 0.5301 & $4.27 E+08$ \\
\hline
\end{tabular}

$i=$ initial

eq $=$ equilibrium

Table 27. $\mathrm{Pb} K d$ Values for $100 \mathrm{mg} / \mathrm{L}$ Lead Ion and Different PH With ETE SOil

Sample $\mathrm{Pb} i, \mathrm{mg} / \mathrm{L}$ [H] i $\mathrm{Pb}$ eq, $\mathrm{mg} / \mathrm{L}$ g Soil $\mathrm{Pb} \mathrm{Kd}$

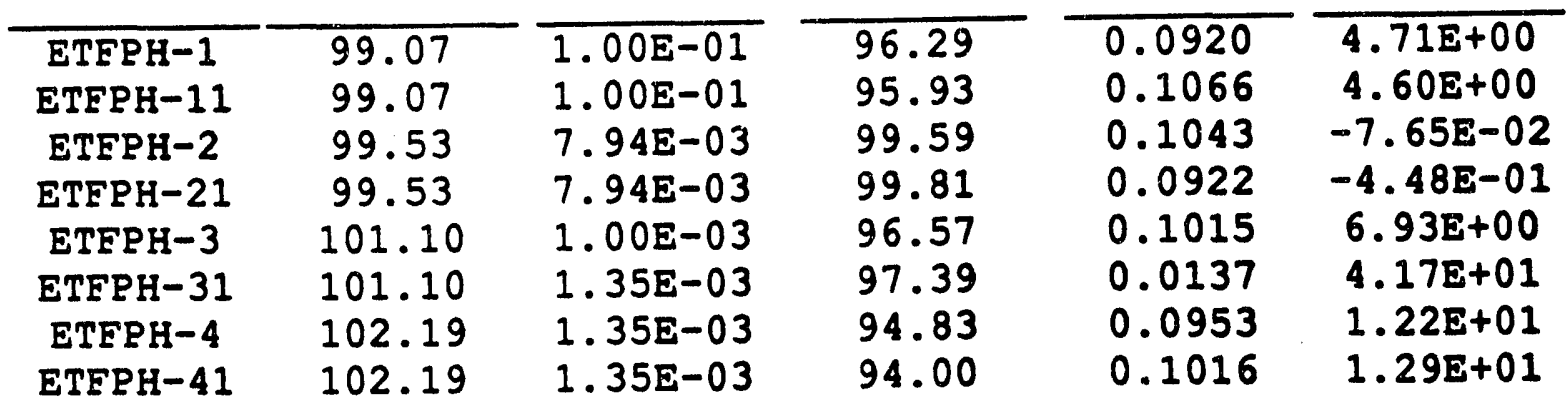

$i=$ initial

eq $=$ equilibrium 
D.L. FISH

WSRC-RP-92-326

March 20, 1992

Page 25 of 45

\subsubsection{So11 Kd's for Cesium Ion, Cst}

Four different concentrations of cesium nitrate solution were contacted with 0.01 gram of each of the soils, 40-60 mesh fraction except for the ETE soll which was not sieved. These solutions, lM, $0.1 \mathrm{M}, 0.05 \mathrm{M}$, and $0.01 \mathrm{M} \mathrm{CsNO}_{3}$, were contacted with the soils for three days on the shaker. Results of the $\mathrm{Kd}$ determinations appear in Table 28 .

Table 28. Kd Values for $1 \mathrm{M}, 0.1 \mathrm{M}, 0.05 \mathrm{M}$, and $0.01 \mathrm{M}$ Cesium Ion with Each Soil

\begin{tabular}{|c|c|c|c|c|}
\hline Sample & Cs $i, \mathrm{mg} / \mathrm{L}$ & $\mathrm{Cs}, \mathrm{eq}, \mathrm{mg} / \mathrm{I}$ & 9 Soil & Cs $\mathrm{Kd}, \mathrm{mL} / \mathrm{g}$ \\
\hline $\begin{array}{l}\text { Tr1 so11 10-6 } \\
\text { TF1-Cs1M } \\
\text { TE1-Cs0.1M } \\
\text { TE1-Cs0.05M } \\
\text { TE1-Cs0.01M }\end{array}$ & $\begin{array}{c}\text { mesh } \\
120808 \\
9756 \\
5946 \\
1235\end{array}$ & $\begin{array}{c}120573 \\
10807 \\
5271 \\
998\end{array}$ & $\begin{array}{l}0.1003 \\
0.1008 \\
0.1009 \\
0.1045\end{array}$ & $\begin{array}{c}2.91 E-01 \\
-1.45 E+01 \\
1.90 E+01 \\
3.41 E+01\end{array}$ \\
\hline $\begin{array}{l}\text { TY2 So11 10- } \\
\text { TE2-Cs1M } \\
\text { TE2-Cs0.1M } \\
\text { TE2-Cs } 0.05 \mathrm{M} \\
\text { TE2-Cs0.01M }\end{array}$ & $\begin{array}{l}\text { mosh } \\
120808 \\
9756 \\
5946 \\
1235\end{array}$ & $\begin{array}{c}87498 \\
10833 \\
4932 \\
985\end{array}$ & $\begin{array}{l}0.1009 \\
0.1040 \\
0.1003 \\
0.1029\end{array}$ & $\begin{array}{c}5.66 \mathrm{E}+01 \\
-1.43 \mathrm{E}+01 \\
3.07 \mathrm{E}+01 \\
3.70 \mathrm{E}+01\end{array}$ \\
\hline $\begin{array}{l}\text { TwX Soil } 40-6 \\
\text { TNX-Cs1M } \\
\text { TNX-Cs0.1M } \\
\text { TNX-Cs0.05M } \\
\text { TNX-Cs0.01M }\end{array}$ & $\begin{array}{c}\text { mosh } \\
120808 \\
9756 \\
5946 \\
1235\end{array}$ & $\begin{array}{c}125499 \\
12744 \\
5431 \\
1069\end{array}$ & $\begin{array}{l}0.1000 \\
0.1004 \\
0.1047 \\
0.1019\end{array}$ & $\begin{array}{c}-5.61 E+00 \\
-3.50 E+01 \\
1.36 E+01 \\
2.29 E+01\end{array}$ \\
\hline $\begin{array}{l}\text { ETY SOL1 } \\
\text { ETF1-Cs1M } \\
\text { ETE2-Cs1M } \\
\text { ETF1-Cs0.1M } \\
\text { ETE2-Cs0.1M } \\
\text { ETE1-Cs0.05M } \\
\text { ETE2-Cs0.05M } \\
\text { ETE1-Cs0.01M } \\
\text { ETE2-Cs0.01M }\end{array}$ & $\begin{array}{c}120808 \\
120808 \\
9756 \\
9756 \\
5946 \\
5946 \\
1235 \\
1235\end{array}$ & $\begin{array}{c}123089 \\
121266 \\
11942 \\
11827 \\
5634 \\
5778 \\
1191 \\
1176\end{array}$ & $\begin{array}{l}0.1098 \\
0.1096 \\
0.1090 \\
0.1059 \\
0.1006 \\
0.1098 \\
0.1055 \\
0.1062\end{array}$ & $\begin{array}{c}-2.53 E+00 \\
-5.17 E-01 \\
-2.52 E+01 \\
-2.48 E+01 \\
8.26 E+00 \\
3.97 E+00 \\
5.25 E+00 \\
7.09 E+00\end{array}$ \\
\hline
\end{tabular}

$i=$ initial

eq $=$ equilibrium 
D.I. FISH

WSRC-RP-92-326

March 20, 1992

Page 26 of 45

\subsubsection{Oranyl Ion, $\mathrm{UO}_{2}{ }^{2+}, \mathrm{Kd}^{\prime}$ for Iach Soll}

The Kd values observed for sorption of uranyl ion from a $0.01 M$ uranyl nitrate solution at $\mathrm{pH} 3$ on 40-60 mesh samples of TF1, TF2, and TNX soils and the unsieved ETF soil are found in Table 29.

Table 29. Kd Values for Uranyl Ion With Each Soil $(\mathrm{pH}=3)$

\begin{tabular}{|c|c|c|c|c|}
\hline Sample & $U i, \mathrm{mg} / \mathrm{L}$ & $\mathrm{U}, \mathrm{eq}, \mathrm{mg} / \mathrm{L}$ & g soil & $\mathrm{U} \mathrm{Kd}, \mathrm{mL} / \mathrm{g}$ \\
\hline $\begin{array}{ll}\text { TE1 } & \text { Soil } \\
\text { TE2 } & \text { Soil } \\
\text { TNX } & \text { Soil } \\
\text { ETE Soil }\end{array}$ & $\begin{array}{l}2245 \\
2245 \\
2245 \\
2245\end{array}$ & $\begin{array}{l}2168 \\
2211 \\
2108 \\
2151\end{array}$ & $\begin{array}{l}0.1005 \\
0.1004 \\
0.1002 \\
0.1027\end{array}$ & $\begin{array}{l}5.30 E+00 \\
2.30 E+00 \\
9.73 E+00 \\
6.38 E+00\end{array}$ \\
\hline
\end{tabular}

\subsubsection{S1116e sand Ird'}

The results of contacting silica sand with solutions of $\mathrm{UO}_{2}{ }^{2+}$, $\mathrm{Hg}^{2+}, \mathrm{Pb}^{2+}$, and $\mathrm{Cs}^{+}$are found in Table 30 .

Table 30. Kd Values for four Ions, $\mathrm{UO}_{2}{ }^{2+}, \mathrm{Hg}^{2+}, \mathrm{Pb}^{2+}$, and $\mathrm{Cs}^{+} \mathrm{Wlth}$ silica sand

Contacting

solution

g.sand Metal 1. ma/rt

Metal eq $\mathrm{mo} / \mathrm{L}^{*} \mathrm{Kd} \mathrm{mL} / \mathrm{g}$

$\mathrm{CsNO}_{3}$

0.2033

$8.08 \mathrm{E}-3 \mu \mathrm{Cl} / \mathrm{mL}$

$8.50 \mathrm{E}-3 \mu \mathrm{C} 1 / \mathrm{mL}$

$-7.17 E+00$

$\mathrm{UO}_{2}\left(\mathrm{NO}_{3}\right)_{2}$

0.2017

2173

2226

$-1.77 E+00$

$\mathrm{Hg}\left(\mathrm{NO}_{3}\right)_{2}$

0.2010

5.69

4.94

1. 13E+01

$\mathrm{Pb}\left(\mathrm{NO}_{3}\right)_{2}$

0.2037

2.19

1.50

3. $44 E+01$

* Units except for Cs data which was analyzed by $\boldsymbol{\gamma}$-counting

1 = initial metal concentration

eq - equilibrium metal concentration 
D.I. EISH

WSRC-RP-92-326

March 20, 1992

Page 27 of 45

\subsection{Soll Capacities and Isotherms}

Isotherms ior mercury and lead were plotted for most of the soils.

\subsubsection{IFI Soil Isotherms}

\section{Hg lsotherms for TF1 Soll - 2 Mesh Sizes}

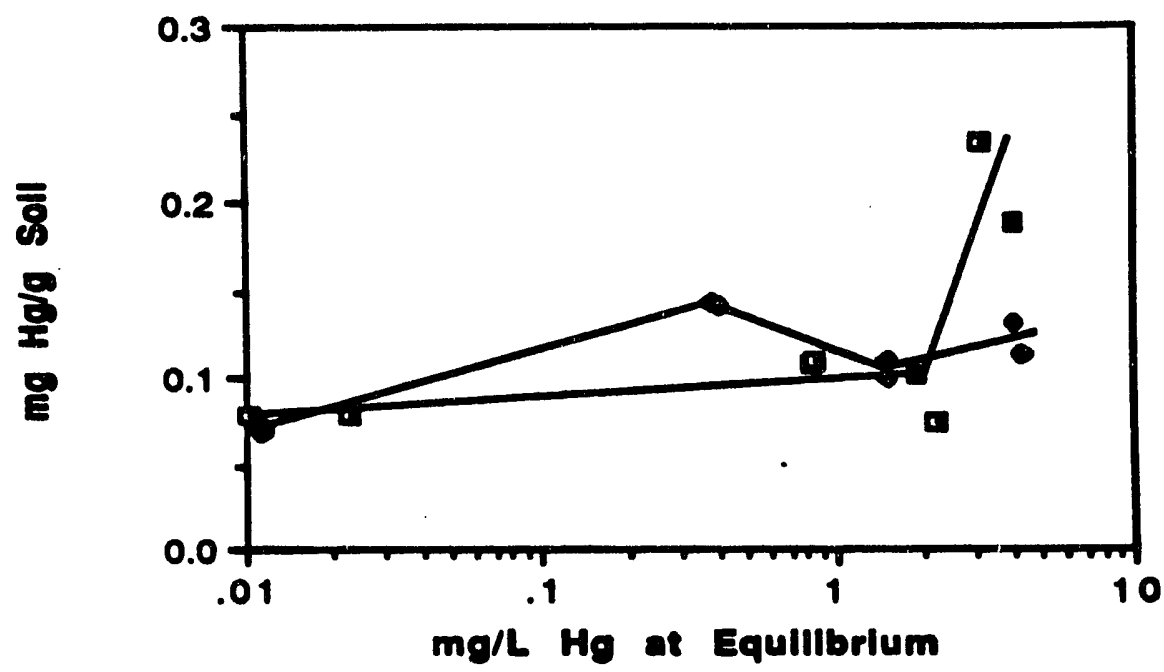

- 40-80 meah

- 80-100 mosh

rigure 2. $\mathrm{Bg}^{2+}$ on $1 \mathrm{TP}$ Sol10

Pb Isotherm for TF1 Soll

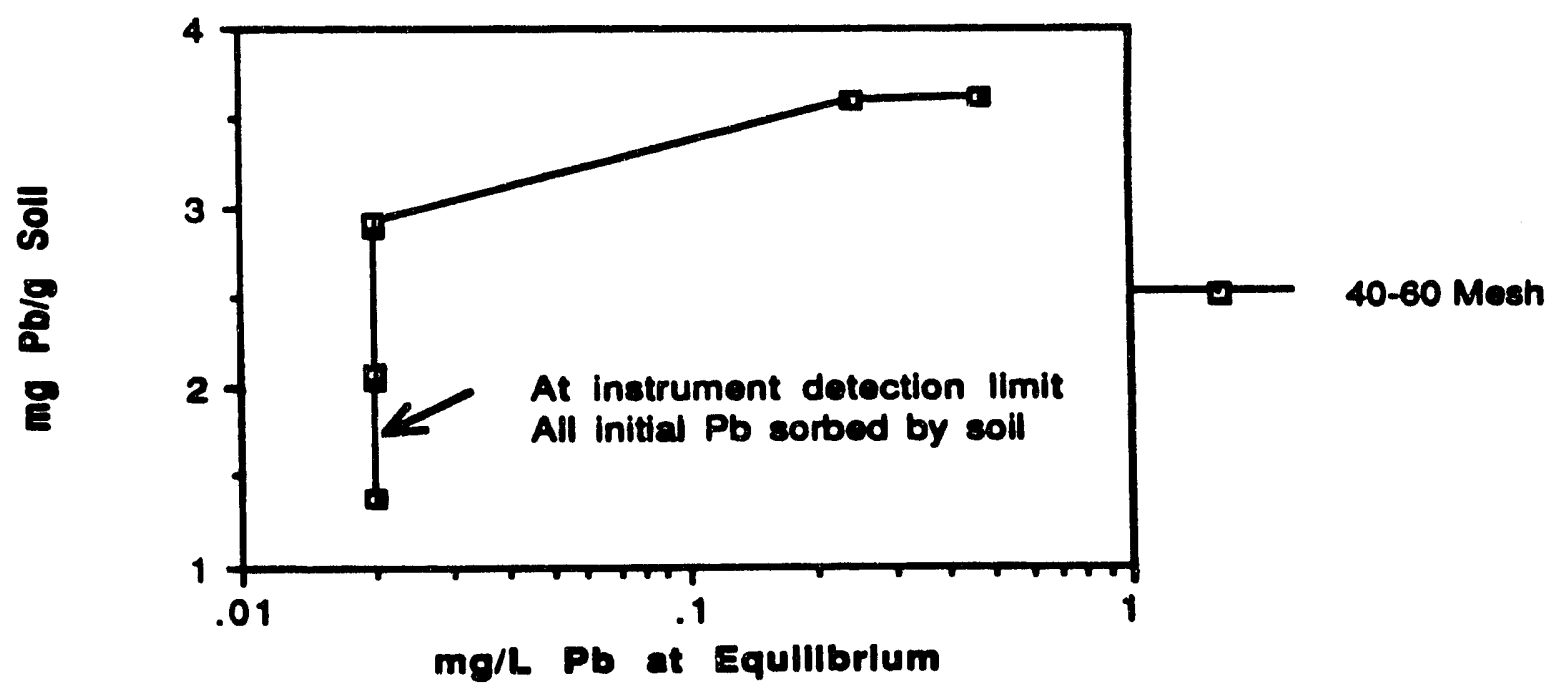

Igure 3. $P b^{2+}$ on TrI solls 
D.L. EISH

WSRC-RP-92-326

March 20, 1992

Page 28 of 45

4.4.2 TI2 s011 Isotherms

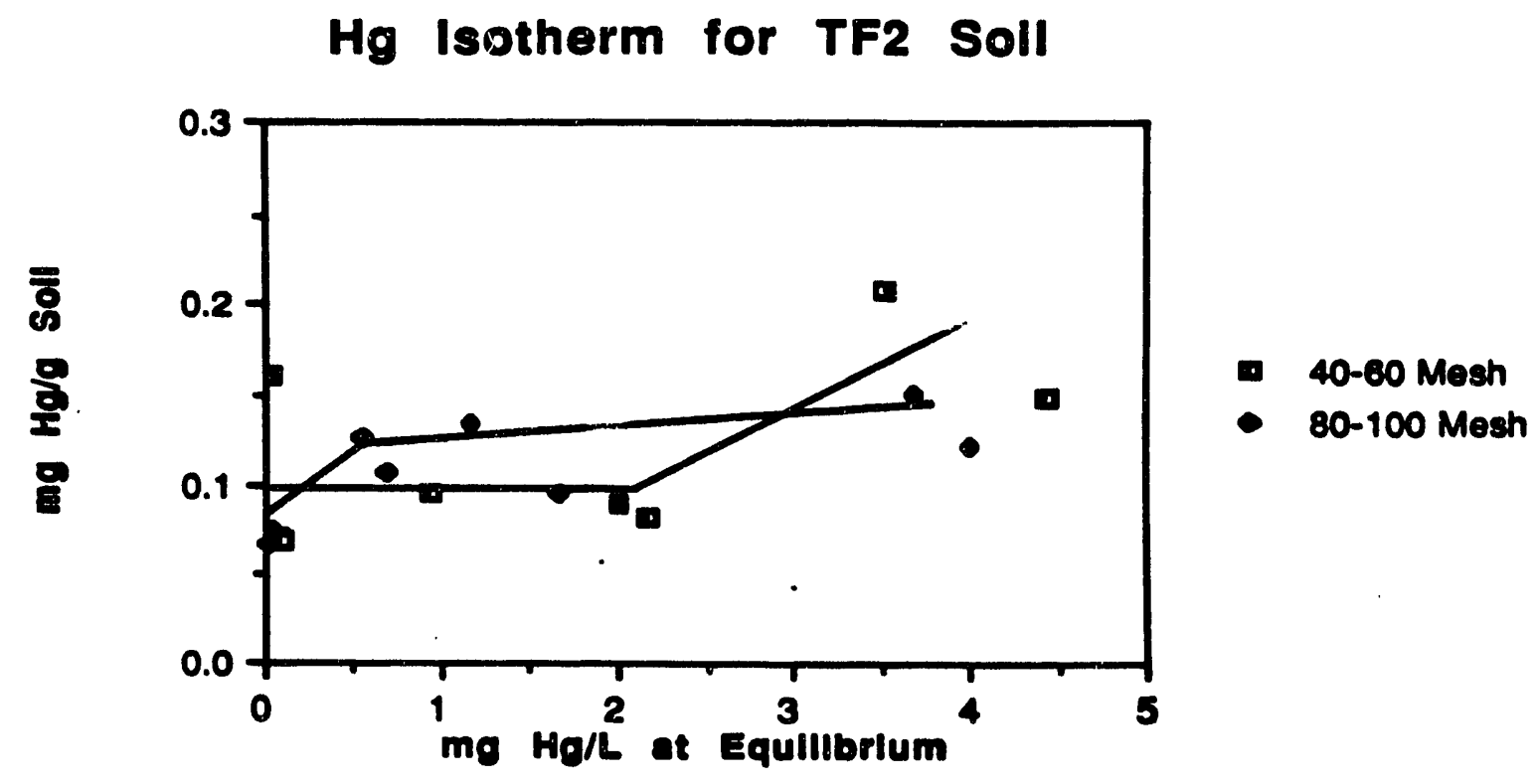

Iigure 4. $\mathrm{Eg}^{2+}$ on Tr2 Sol1s

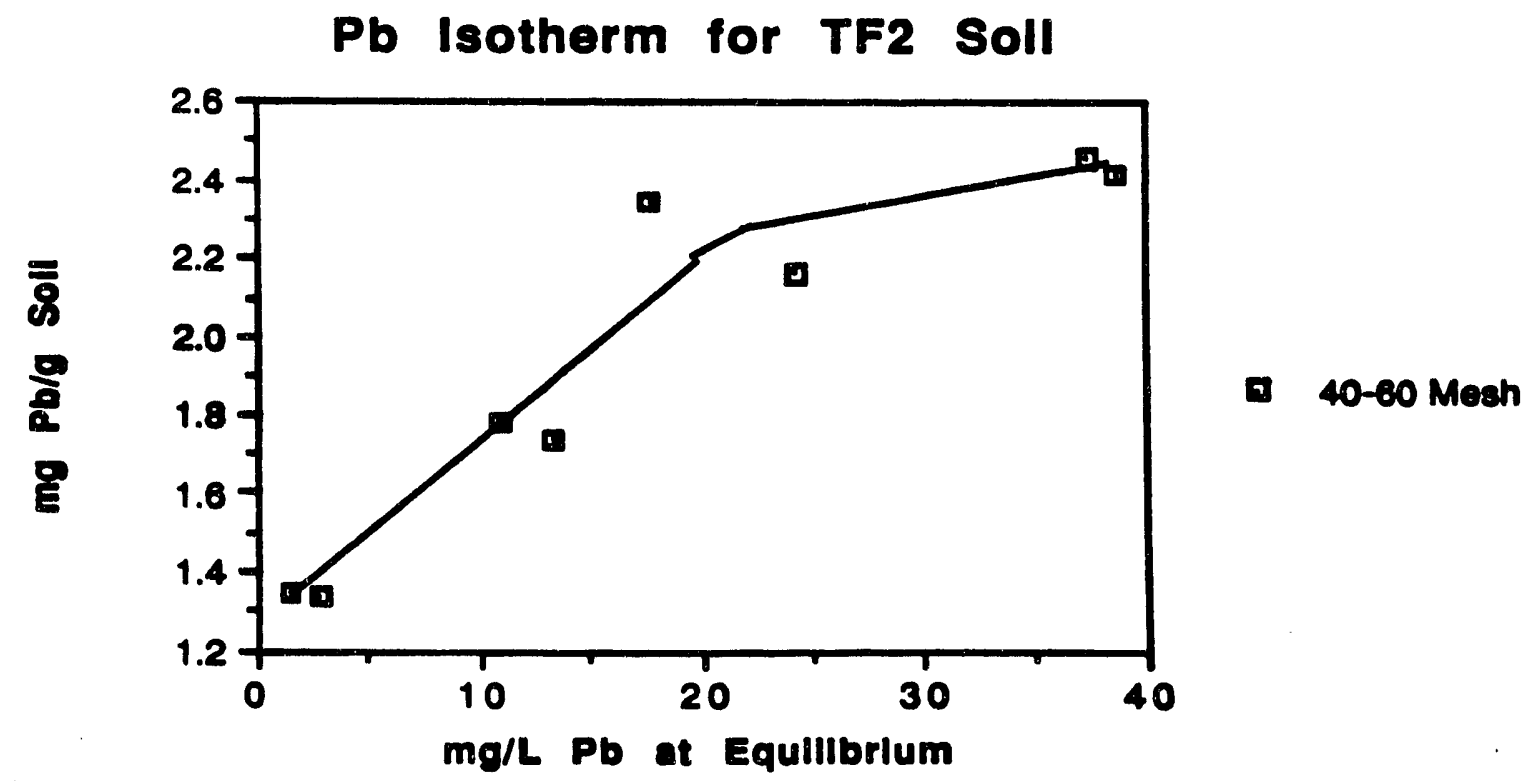

Ilgure 5. $\mathrm{Pb}^{2+}$ on $2 \mathrm{Tr}$ so11 
D.L. EISH

WSRC-RP $-92-326$

March 20, 1992

Page 29 of 45

\subsubsection{TwX soil Isotherms}

Only the mercury isotherms for TNX solls have significance. Lead ion was sorbed so well by this soll that equilibrium concentrations were below the detection limit of the ICPES instrument for nearly every sample (Table 22).

Hg lsotherm for TNX Soll

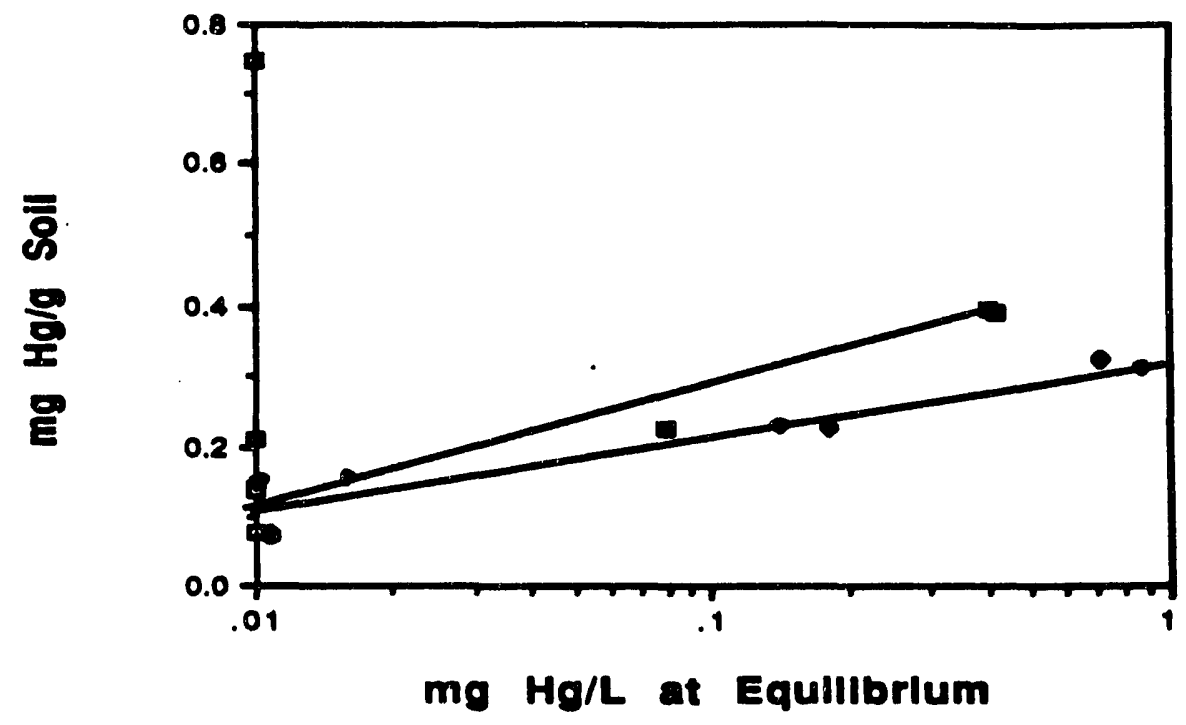

- 40-80 moen

- 80-100 moen

Iigure 6. $\mathrm{Bg}^{2+}$ on Tux so11

\section{4 .3 EW soil Isotherm}

Hg Isothorm for ETF Soll

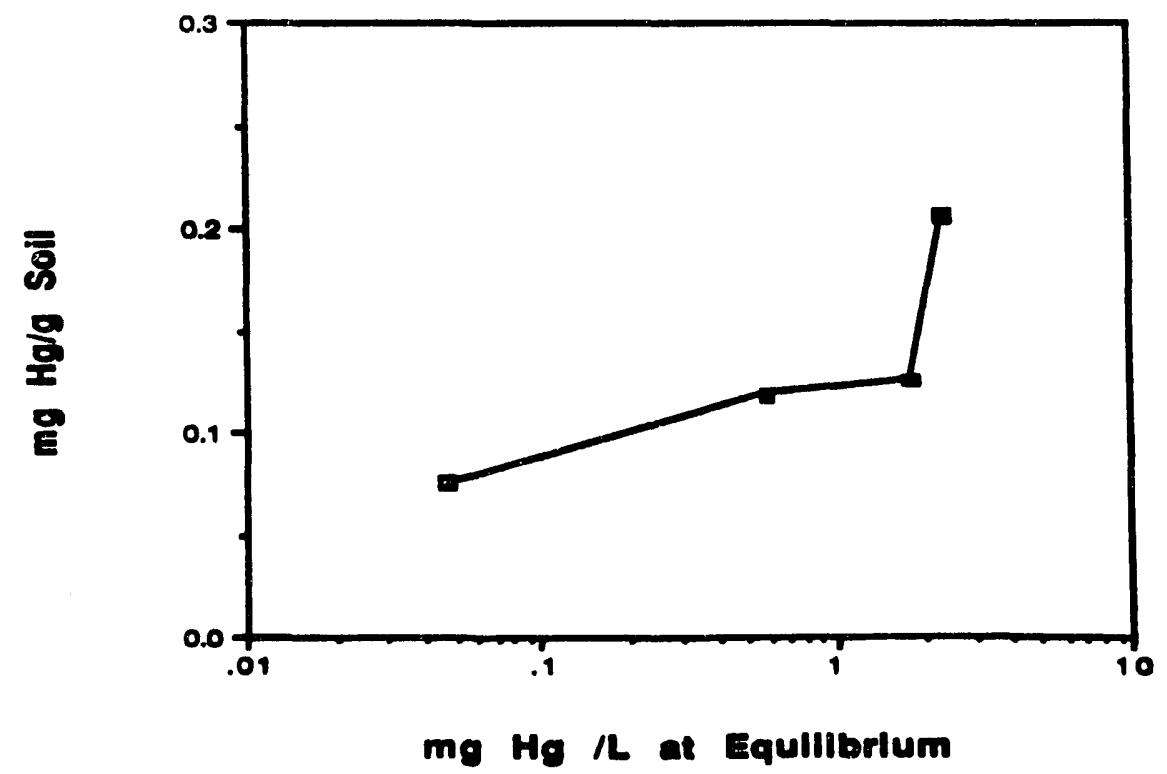

rigruxe7. Morcurie ton on IrT Soll 
As in the case of TNX soil, the ETF soll sorbed $\mathrm{Pb}^{2+}$ so well that the equilibrium ion concentration was less than the detection limit of the instrument. (Table 19)

\subsection{A Tabulation of Ion Exchanging Species}

Table 31 contains data collected in an attempt to establish a mass balance among ion exchanging species in solution and on the soil. It was presumed that $\mathrm{Pb}^{2+}$ or $\mathrm{Hg}^{2+}$, and possibly $\mathrm{H}^{+}$in solution would exchange for $\mathrm{Na}^{+}$in the kaolinite clay of the solls.

Table 31. A Comparison of Milliequivalents of Ions Exchanged Between Soll and Solution for $100 \mathrm{mg} / \mathrm{L} \mathrm{Pb}^{2+}$ at Four $\mathrm{pH}^{\prime} \mathrm{s}$

\begin{tabular}{|c|c|c|c|c|}
\hline SAMPLE & Solution pH & $\begin{array}{l}\text { meq Na change } \\
\text { per g soil }\end{array}$ & $\begin{array}{l}\text { mag Pb change } \\
\text { per g soil }\end{array}$ & $\begin{array}{l}\text { meq i change } \\
\text { per a soil }\end{array}$ \\
\hline TF1-1 & 0.45 & $7.44 \mathrm{E}-03$ & $-2.16 \mathrm{~B}-03$ & $9.62 \mathrm{E}+00$ \\
\hline TF1-11 & 0.45 & $7.60 \mathrm{~B}-03$ & $-1.73 \mathrm{~B}-03$ & $1.30 \mathrm{~B}+01$ \\
\hline TF1-2 & 1.51 & $8.12 \mathrm{E}-03$ & $-4.21 \mathrm{~B}-03$ & $3.77 \mathrm{~B}+00$ \\
\hline TE1-21 & 1.51 & $8.01 \mathrm{E}-03$ & $-4.77 \mathrm{E}-03$ & $1.97 \mathrm{~B}+00$ \\
\hline TF1-3 & 2.78 & $7.37 \mathrm{~B}-03$ & $-2.92 B-02$ & $-1.07 \mathrm{E}-01$ \\
\hline TE1-31 & 2.78 & $1.59 \mathrm{~B}-02$ & $-3.08 \mathrm{~B}-02$ & $-1.13 \mathrm{~B}-01$ \\
\hline TF1-4 & 4.61 & $6.16 \mathrm{~B}-03$ & $-7.20 \mathrm{E}-02$ & $-1.70 \mathrm{E}-02$ \\
\hline TE $1-41$ & 4.61 & $5.80 \mathrm{E}-03$ & $-6.73 \mathrm{~B}-02$ & $-1.60 \mathrm{~B}-02$ \\
\hline TF2-1 & 0.45 & $4.43 E-03$ & $-2.54 \mathrm{~B}-03$ & $1.49 \mathrm{E}+01$ \\
\hline TF2-11 & 0.45 & $4.49 E-03$ & $-2 \cdot 31 \mathrm{~B}-03$ & $1.88 \mathrm{E}+01$ \\
\hline TF2-2 & 1.51 & $5.14 E-03$ & $-3.24 E-03$ & $3.63 \mathrm{E}+00$ \\
\hline TF2-21 & 1.51 & $5.74 \mathrm{E}-03$ & $-3.35 E-03$ & $-1.56 \mathrm{E}-01$ \\
\hline TF2-3 & 2.78 & $4.48 E-03$ & $-1.04 B-02$ & $-5.64 B-02$ \\
\hline TF2-31 & 2.78 & $4.48 E-03$ & $-1.01 \mathrm{~B}-02$ & $-5.71 \mathrm{~B}-02$ \\
\hline TE2-4 & 4.61 & $3.66 \mathrm{E}-03$ & $-4.71 B-02$ & $-1.44 \mathrm{~B}-03$ \\
\hline $\operatorname{TE2}-41$ & 4.61 & $3.39 \mathrm{~B}-03$ & $-4.61 B-02$ & $-1.43 \mathrm{~B}-03$ \\
\hline TWX-1 & 0.45 & $6.45 E-02$ & $0.00 \mathrm{E}+00$ & $1.75 \mathrm{E}+01$ \\
\hline THX -11 & 0.45 & $7.21 \mathrm{~B}-02$ & $0.00 \mathrm{E}+00$ & $1.28 B+01$ \\
\hline THX -2 & 1.51 & $8.36 \mathrm{~B}-02$ & $-1.76 \mathrm{~B}-03$ & $2.35 \mathrm{E}+00$ \\
\hline TNX-21 & 1.51 & $8.36 \mathrm{~B}-02$ & $-1.60 \mathrm{~B}-03$ & $1.18 \mathrm{~B}+00$ \\
\hline TNX - 3 & 2.78 & $8.26 \mathrm{~B}-02$ & $-4.46 B-02$ & $-9.93 \mathrm{~B}-03$ \\
\hline TNX-31 & 2.78 & $9.17 \mathrm{~B}-02$ & $-4.84 \mathrm{E}-02$ & $-1.01 \mathrm{~B}-02$ \\
\hline TNX-4 & 4.61 & $6.54 \mathrm{E}-02$ & $-7 \cdot 18 \mathrm{~B}-02$ & $-1.63 \mathrm{~B}-02$ \\
\hline$T N X-41$ & 4.61 & $7.35 \mathrm{~B}-02$ & $-6.73 B-02$ & $-1.628-03$ \\
\hline
\end{tabular}

A - signifies lost from solution concentration-sorbed by soll

A + signifies gained by solution - lost from soil 
D.L. EISH

WSRC-RP $-92-326$

March 20, 1992

Page 31 of 45

Table 32. A Comparison of Milliequivalents of Ions Exchanged Between Soil and Solution for $\mathrm{Pb}$ Ion in the Presence of $1 \mathrm{M} \mathrm{Ca}$ Ion

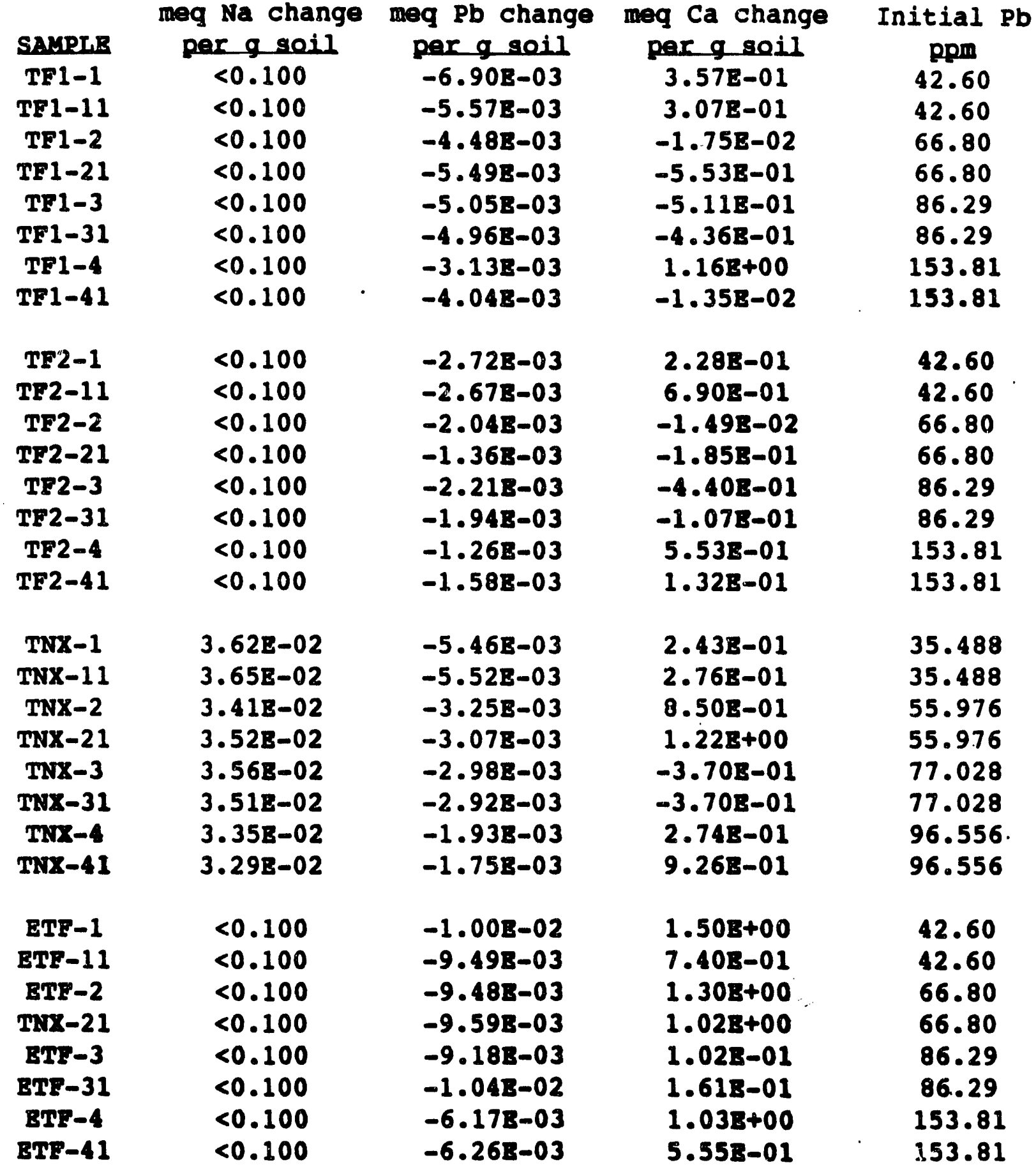


In Table 32., a (-) number signifies concentration lost from solution to the soil and $a(+)$ number signifies concentration lost from the soil to the solution.

Samples of TF1, TF2, and TRX soils were shaken with detonized water to determine if $\mathrm{B}^{+}$concentrations in solution would change in the absence of $\mathrm{Pb}^{2+}$. The results of this experiment are given in Table 33.

Table 33. The Change in Solution Eydrogen Ion Concentration on Contacting Three Solls With Delonized Water.

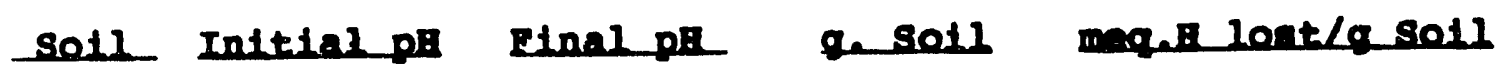

\begin{tabular}{lllll} 
TF1 & 5.69 & 6.35 & 1.0292 & $1.571-6$ \\
TF2 & 5.69 & 6.10 & 1.0060 & $1.248-6$ \\
THX & 5.69 & 6.13 & 1.0299 & $1.26 \mathrm{~B}-6$ \\
\hline
\end{tabular}

Samples of the same solls were also shaken with delonised water which had been $\mathrm{pB}$ adjusted to $4.11 \mathrm{deing} \mathrm{BmO}_{3}$. The anonnt of $\mathrm{Ma}^{+}$ liberated from the soils by the hydrogen fon 1 s found for ach soll in Table 34 .

Table 34. The Change in Solution Sodium Ion Concentration on Contacting Three Solls With pa 4.11 Water

Soll maqria denorbed/g sotl gesoll magellaorbad/g soll

$\begin{array}{llll}\text { T21 } & 3.61 \mathrm{E}-3 & 1.0282 & 3.61 \mathrm{E}-3 \\ \mathrm{TR2} & 1.47 \mathrm{E}-3 & 1.0160 & 1.47 \mathrm{E}-3 \\ \text { TIX } & 2.62 \mathrm{E}-3 & 1.0229 & 2.26 \mathrm{E}-3\end{array}$

\subsection{Renoving Ions Already sorbod on solls}

Table 35 detalls the resulto of contacting solle, already containing a known amount of $\mathrm{Pb}^{2+}, \mathrm{Bg}^{2+}, \mathrm{VO}_{2}{ }^{2+}$, and $\mathrm{Ca}^{+}$, with $1 \mathrm{HCa}\left(\mathrm{NO}_{3}\right)_{2}$ and $0.01 \mathrm{M} \mathrm{HOO}_{3}$ in order to test the kinetics of the reverse of the sorption reaction for those lons. 


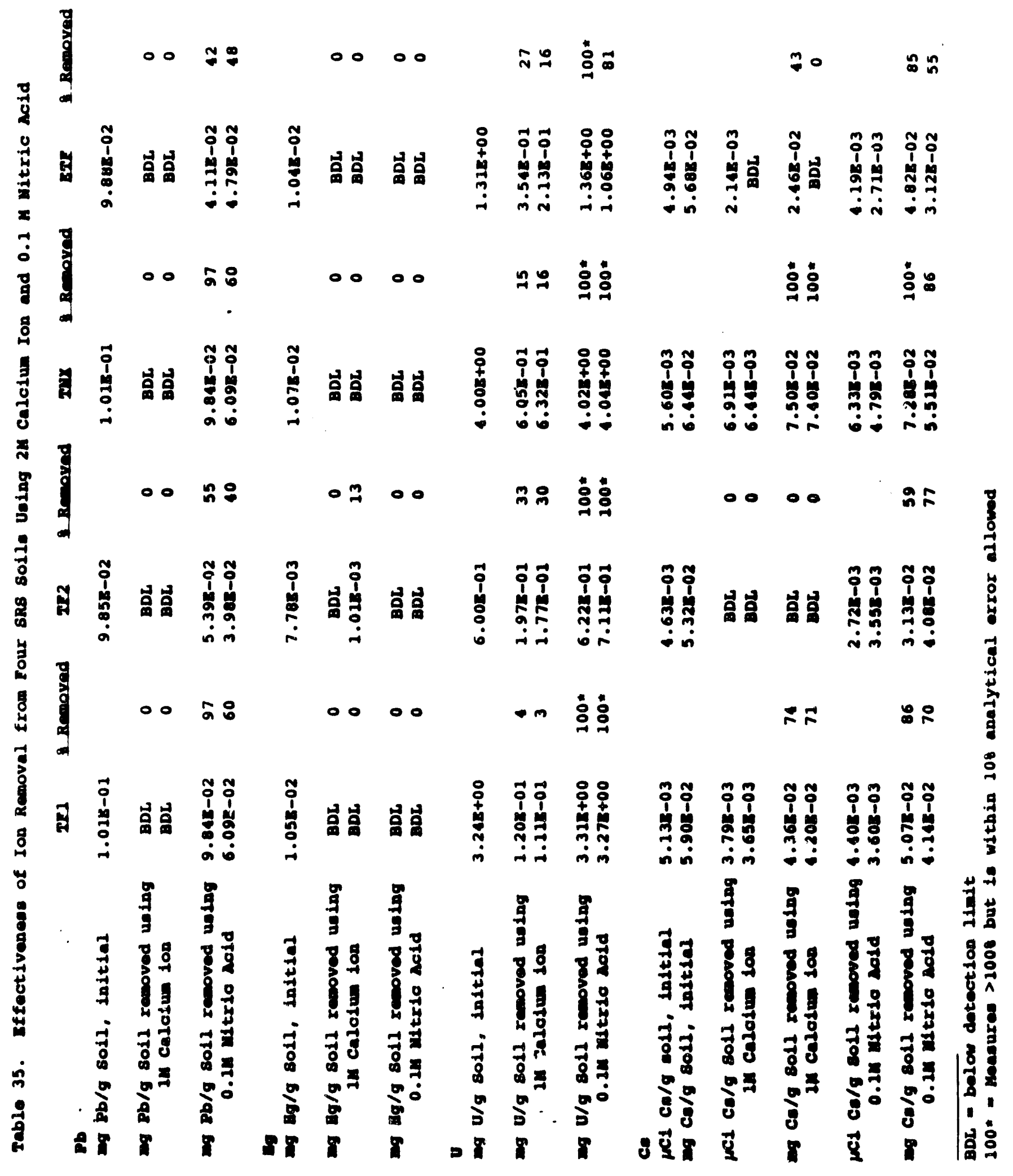




\subsection{DIsCOs8I0n}

It must be remembered that this study was done with actual soils, made as homogeneous as possible by mixing and sieving, rather than with pure kaolinite. Therefore, $\mathrm{Kd's}$ are less for these samples than they would have been for the clay alone. Discrepencles in results between duplicate samples can be attributed, for the most part, to slightly different percentages of clay and sand in the samples.

\subsection{Phyical Boharior of solls in Different Bolutions}

Although the soils were all comprised of mostly kaolinite and sand, thelr swelling behavior in different solutions was not uniform. For example, TF1 and TF2 solls exhibited the greatest swelling (amallest bulk density) at very low pI in the preaence of norcuric 10n, though they tended otherwise to tncrease in wet volum as pl was increased. The volume of the THX soll, on the other hand, was at a minimun (largest bulk deneity) in the sam morcury solution. Conversely, tho uranyl ion, at nearly the sam $\mathrm{pl}$ as the mercuric nitrate solution, had little effect on TF1 soil relative to delonized water but generated a shrinking of TF2 soll and swelling for BTF (not shorm in Figure 8) and Tix solls. The bulk denelty of so11s, thus appears to be related not only to the pl of the solution contacting the soll and the soll itself but also the contacting lon in the solution.

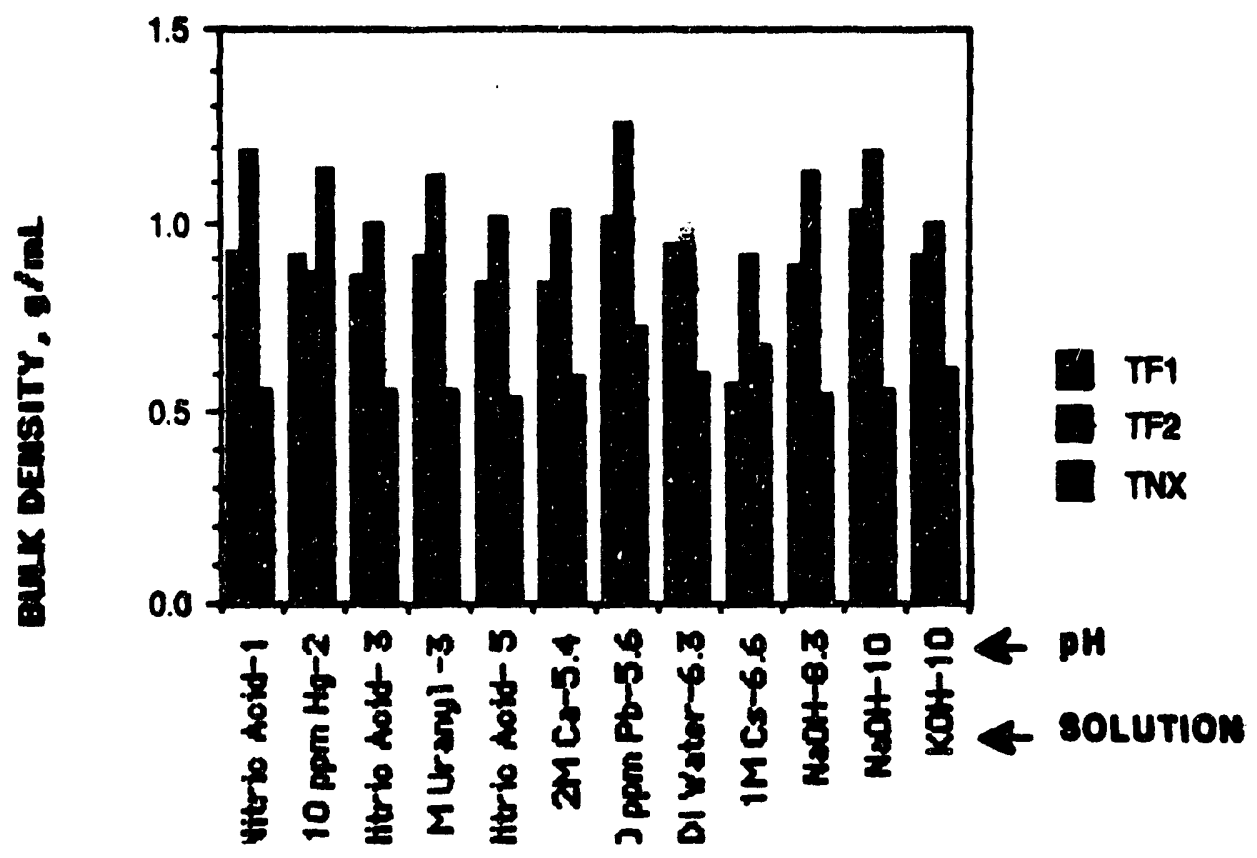

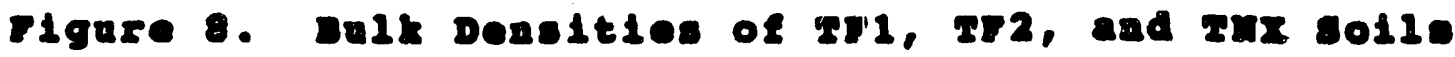




\section{D.I. FISE}

WSRC-RP-92-326

March 20, 1992

Page 35 of 45

Although the X-ray diffraction data did not identify the presence of iron in the soils, the rust colored appearance of TF1, TF2 and the ETF solls would indicate that iron 18, indeed, present. It may exist as an amorphous FeO(OE) spectes that would not generate any peaks in the X-ray spectrum. Or, the concentration of a cryetalline compound may be too small to detect.

\subsection{The Id Values}

Kd values, when an ton was removed from solution by the soll, were positive numbers. When the ion concentration change on contact with the soll was negliglble, the sign of Rd was negative. Very small or negative Id's Indicated little or no attraction for the Ion by the so11.

In general, it may be aaid that the magnitude of the rorption Kd could be controlled for any given metal ion by the concentration of competing 1ons, such as $\mathrm{B}^{+}$or $\mathrm{Ca}^{2+}$. $\mathrm{Kd}^{\prime}$ f for heavy matal lons wore reduced as the concentration of $\mathrm{B}^{+}$or $\mathrm{Ca}^{2+}$ was increased In the same solution because of competition for sorption sites. For example, Id for $\mathrm{Pb}^{2+}$ on $\mathrm{MF} 1$ soll was lowered by a factor of 1,000 in the presence of $1 \mathrm{M} \mathrm{Ca}^{2+}$ and varled fron $8 \mathrm{~s}-1 \mathrm{mr} / \mathrm{g}$ to $z 1 \mathrm{~s}+5 \mathrm{mr} / \mathrm{g}$ at $\mathrm{ps} 0.46$ and 4.6, respectively. F1gure 9. graphically show the differonces in $\mathrm{Pb}^{2+}$ Kd that competing lons can impose on sorption. It also shome.

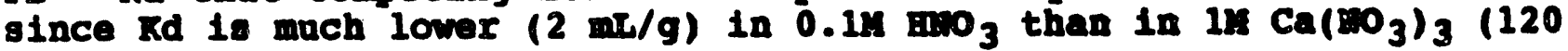
$\mathrm{mr} / \mathrm{g})$. that concentration is not the only paraneter involved. The Law of Mase Action for equilibria ts most certainly at work in

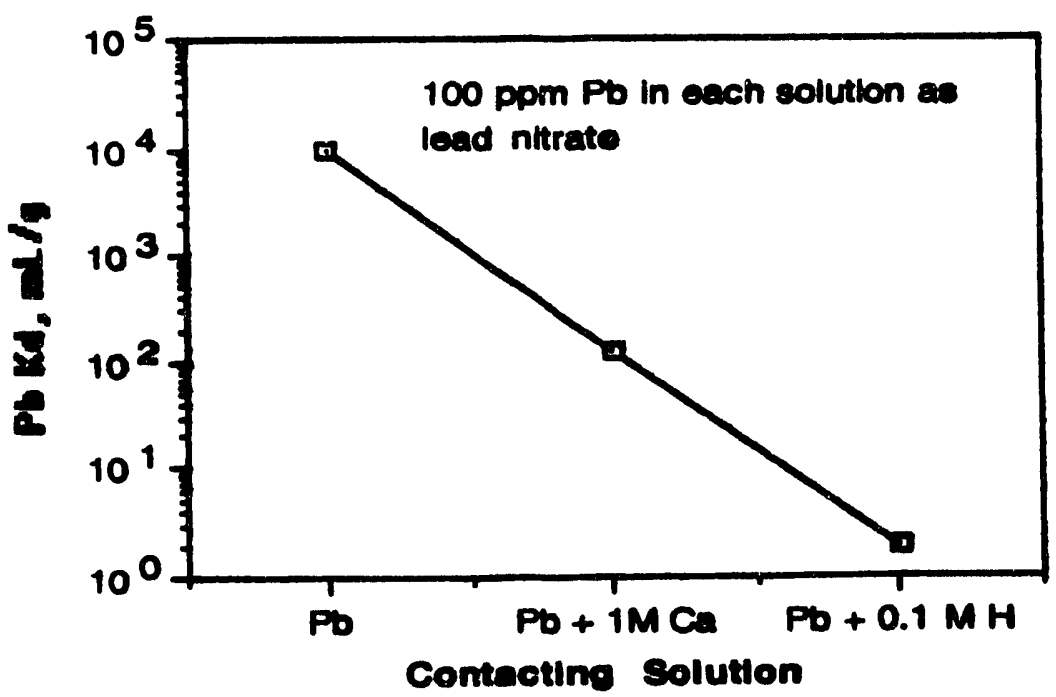

Figure 9. The Elect on $\mathrm{pb}^{2+}$ id N1th Conpetigg Ions Present th1s effect, but other factors such as aeta potent1al, selectivity. 
and swelling of the matrix may also play a role in the ability of the soil to sorb certain 10ns. It ls of interest to note that, even in the presence of a large excess of $\mathrm{B}^{+}$or $\mathrm{Ca}^{2+}$, all four solls showed a selective preference for $\mathrm{Bg}^{2+}$ and $\mathrm{Pb}^{2+}$.

Decreasing the particle size of a soil lncreased 1ts ability to sorb lons from solution. (See Figures 2,4, and 6) This result is expected because increased surface area for smaller particles meane more sorption sites. Bowever, the soll samples of amaller particle size also contained 20-40 percent more kaolinite, the expected primary sorbling component of the so11s, and should exhibit greater capacity and larger $\mathrm{Kd}$ 's for that reason as well. The relationohip between amount of kaolinite and $\mathrm{kd}$ was not linear, however. Kd values did not 1ncrease 20-40 on going fron 10-60 mosh to 80-100 mesh soll particle size.

Kd': measured for pure silica and (Table 31), the other so11 component, showed that the "hard",1.e. sanil, olightly polarizable, lons, $\mathrm{Ca}^{+}$and $\mathrm{OO}_{2}{ }^{2+}$ were not sorbed. The larger, more polarizable, or "goft" Lewts acids 1 , $\mathrm{Eg}^{2+}$ and $\mathrm{Pb}^{2+}$, howerex exhibited $\mathrm{Xd}$ 's of 13 and $34 \mathrm{~m} / \mathrm{g}$, respectively. Such sorption of mallic lone by allica sand is well-documented in the literature.2.3 Th1s etudy did not determine whether the uptake of metal ion on the sand was due to true adsorption on the surface of the sand or to precipitation of an oxyor hydroxy-metal spectes at the solvated surface.

Sorption xd valuee for all four lone were larger with Mix soll than for the other soils. This may, in part, be attributed to the fact that TMX soll was often composed of a lirger percentage of kaolinite than the other solls (Flgure 1). A comparison of $\mathrm{Bg}^{2+} \mathrm{Kd}^{\mathrm{s}}$, however, between 80-100 mesh TP1 soil (65s kaolinite) and 40-60 mesh THX soil (458 kaolinite) shows that the $\mathrm{Mx}$ soll sorbed more morcuric lon at equilibrium than the TFl soll. sinilarly, 40-60 mash THR soil generated larger Id values for $\mathrm{Bg}^{2+}$ than the 80-100 wool TF2 ro11 (50s keolinite). Thus, the anount of kaolinite in the 8011 cannot be the only factor in metal lon uptake by the soll. The Twx soll was the only soll with any residual organic matter associated with it and the added capacity of Tix soll for motal lons 18 attributed to the chelating ability of that organic matter.

Another factor that appeared to have an effect on the Id' for thege solls was the zeta potential, a measure of the not charge on the surface of the soll particles. The 1800 lectric point for $\mathrm{SIO}_{2}$ in nitrate solutions occurs at a pB of about 2.78 .4 raolinito's 1soelectic point is reported to range fro 3.3 to 4.6 .5 In pR- 
regulated experiments in this study, all ions tested $\left(\mathrm{UO}_{2}{ }^{2+}\right.$ was not tested) registered greater $\mathrm{kd}$ values as the $\mathrm{pH}$ increased. This has already been discussed as the result of a competition with $\mathrm{H}^{+}$, but it is important to note that a decided elevation in the $K d$ value occurred at $\mathrm{pH} 3-4$. Figure 10 shows the change in $\mathrm{Pb}^{2}+\mathrm{Kd}$ as a function of $\mathrm{PH}$ for TF1, TF2, and TNX soils.

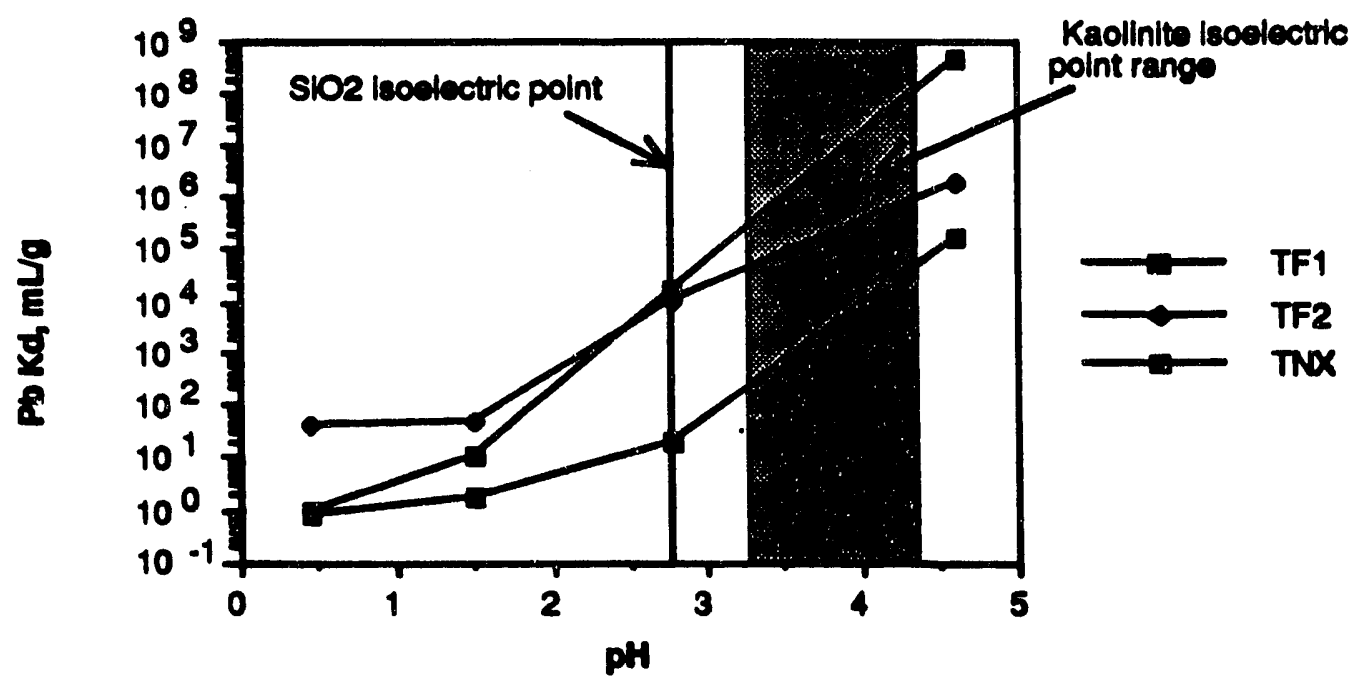

Iigure 10. Pb't Kd for Three solla showing Isoolectric

In the region of the isoelectric point (at the zeta potential), the surface of the soll, which had been positively charged, achieves a net negative charge. This leads to greater attraction for and more sorption of the metal cations onto the surface. It may also lead to precipitation of the cation at the surface. In either case, the ion was removed from solution and became assoclated with the soll. The $\mathrm{pH}$ of the contacting solution can be directly regulated by addition of a known amount of actd or base or it can be indirectly regulated by hydrolysis of salts dissolved in it.

It would appear that sorption of counterions by these solis is also a function of the cation concentration in the contacting solution. The greater the total cation concentration in the solution, the lower the observed value for $\mathrm{Kd}$. Although the $\mathrm{Kd}$ was less, the 1sotherms indicate that, for lead and mercuric lons, the amount of ton sorbed continued to increase with initial metal fon concentration until the soll was saturated. Thls appeared to te the case for the $\mathrm{Cs}^{+} \mathrm{Kd}^{\prime} \mathrm{s}$ for all of the solls. Kd values for the more concentrated $1 M$ and $0.1 \mathrm{M}$ solutions were negative whereas the more dilute $0.05 \mathrm{M}$ and $0.01 \mathrm{M}$ solutions were positive. But, no sorption of cesium ton occurred at 
D.I. FISE
WSRC-RP-92-326

March 20,1992

Page 38 of 45

the two higher solution concentrations. A rationale suggested for that observation 18 that, for the more concentrated $\mathrm{Cs}^{+}$solutions, the surface of the soil may have become quickly saturated with a small amount of counterion, screening any further sorption.

\subsection{Ion Exchange}

Raolinite is not known as a good ion exchanger. Studles with lead Ion (Tables 32 and 33 ) Indicate that $\mathrm{Na}^{+}$1s readily exchanged for $\mathrm{B}^{+}$, though the milliequivalents of $\mathrm{Ha}^{+}$released did not equal the millequivalents of $\mathrm{Pb}^{2+}$ nd $\mathrm{B}^{+}$sorbed. Further, the arount of $\mathrm{Ma}^{+}$

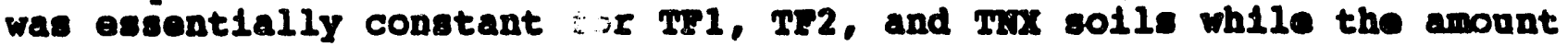
of $\mathrm{Pb}^{2+}$ sorbed varied as a function of solution $\mathrm{pH}$. Then a large amount of $\mathrm{Ca}^{2+}$ was present initislly, the amount of $\mathrm{Pb}^{2+}$ sorbed was constant. Measurable $\mathrm{Na}^{+}$given up by soll was noted only for the Trx samples. It is expected that this sodiun ion was primarily associated with the organic matter in the Tix soil rather than with the clay portion. TF1 and TF2 solis did liberate small quantitioe of $\mathrm{Na}^{+}$in weakly acidic delonized water, and that small arount may have been difficult to detect in the presence of $1 \mathrm{H} \mathrm{Ca} \mathrm{Ca}^{+}$. The $\mathrm{Ma}^{+}$ liberated did not result from dissolution of the soll.

\section{Dosorption}

Equilibrium sorption studies do not generate data that can be reproduced for the reverse, desorption, process. The four lons studied can be easily kept from sorbing on the solls by decreasing the $\mathrm{pB}$ or adding $\mathrm{Ca}^{2+}$ Ion. Using $\mathrm{B}^{+}$or $\mathrm{Ca}^{2+}$ to remove lone already sorbed on the solls showed that only $\mathrm{B}^{+}$was effective and it was efflclent for $\mathrm{OO}_{2}{ }^{2+}$ and $\mathrm{Cs}^{+}$only (Table 35 ).

\section{0 conczusions}

Results of this study indicate that sorption of lone by these solls is not accopplished by a single machanise. soveral sorption processes appeared to be simulatneously at work, those being adsorption, chelation (for TrX so11), and a sall arount of lon exchange. Regulating these processes ware competing lon and their concentrations, surface area, $\mathrm{pH}$, the nature of the sorbing 1ons, and the presence of organic matter. Although it was not addresed in this study, the effect of Iron oxides or 1ron oxyhydroxides present in som solls may also be significant.

Sorption of mercuric and lead lons by the hydrated sand in these solls abould not be 1gnored. This Indicetes that the sand cannot be coneldered uncontaninated after exposure to solutions of certaln motal cations. 


$$
\text { D.L. FISE }
$$

HSRC-RP-92-326

March 20,1992

Page 39 of 45

The use of equilibrium data from $\mathrm{Kd}$ experiments should not be used to predict the desorption of lons from these solls. The kinetics of the reverse of the sorption processes must be understood before any a priorl predictions can be made.

\section{0 gosiges novennce}

All dats fron this study are recorded in $78 R C-\mathrm{NB}-90-345$ and W8RC-MB90-346. Standard solutions were prepared with reagent grade chenicale to chock analytical mothods. Roagent grade chenicale were used for all pa adjuetemets. Analyses wero perfornad by the SRI Analytical Developiont section (AD8) for the following "customer asaleted" analyseas garna counting for Cs-137, cold vapor atoulc absorption for mercury and cesiun, total uranim, and inductively coupled planes atonic enlesion opectrontry for lead, calciun, and sodiu. An Orion pa beter, Hodel 7011, was uecd to manuro bydrogen lon concontration.

\section{- .0 Repranere}

1. R.G. Pearson, Journal of the Anorican Chateal soctety, Os, 3533 (1963).

2. S.F. Relyea and R.J. S1lva, "Application of a sito-binding slectrifal, Double-Iayer Model to meclear Wasto Dipposal". PuL-3898, Eoptember 1981.

3. A.T. Jakublck and I, Fahl, "Ilntte to the one of Tranonraniw Sorption Data in Waste Higration Analyeen". Proccedinge of the Sypostum on Naste Management, Maste Managenont '82, Tucson, Arizona, March 8-11, 1982.

4. G.A. Parks, "The Isoelectric Polnte of solid Oxidee, solid Bydroxides, and squeous Bydroxide 8yotem", Chen. Bov.. 65, 1965, p 177-198.

5. G.A. Parke, "Aqueoue surface Chentetry of Oxtden and Complex oxide utinorale; Egullibrium Concopts in Batural Wator syatemo. Idvances in Chentstry seriee, 67, R.8. Gould, od.. Anorican Chenical soclety Publishera, Mashington D.C.. 1967. p 121-160. 
D.L. EISH

WSRC-RP-92-326

March 20, 1992

Page 40 of 45

\section{APPENDIX 1}

X-RAY SPECTRA FOR FOUR SRS SOILS 
D.I. EISH

WSRC-RP-92-326

March 20, 1992

Page 41 of 45

SPECTRUM EOR TEI SOIL

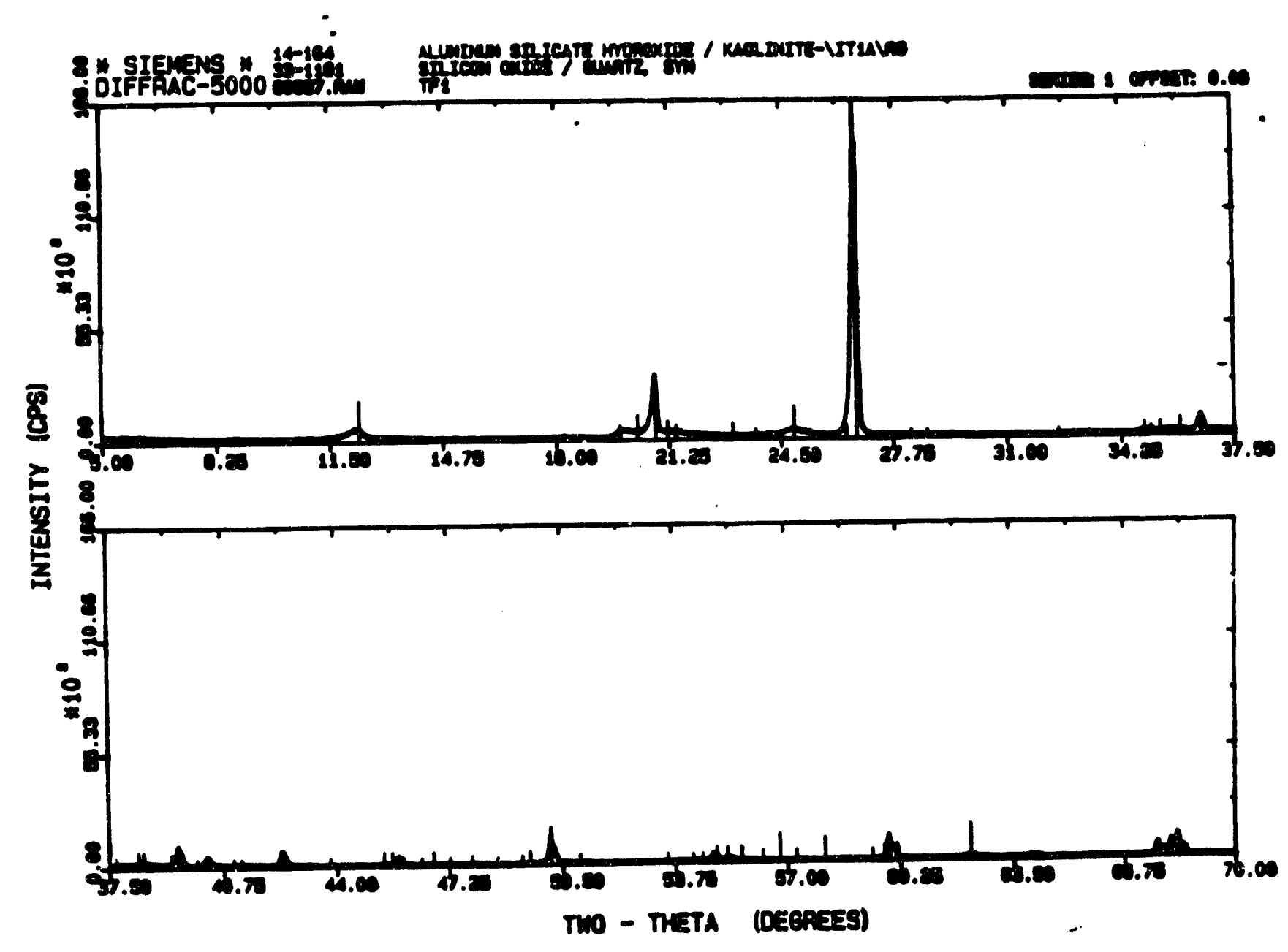


D.L. FISH

WSRC-RP-92-326

March 20, 1992

Page 42 of 45

SPECTRUM EOR TE2 SOIL

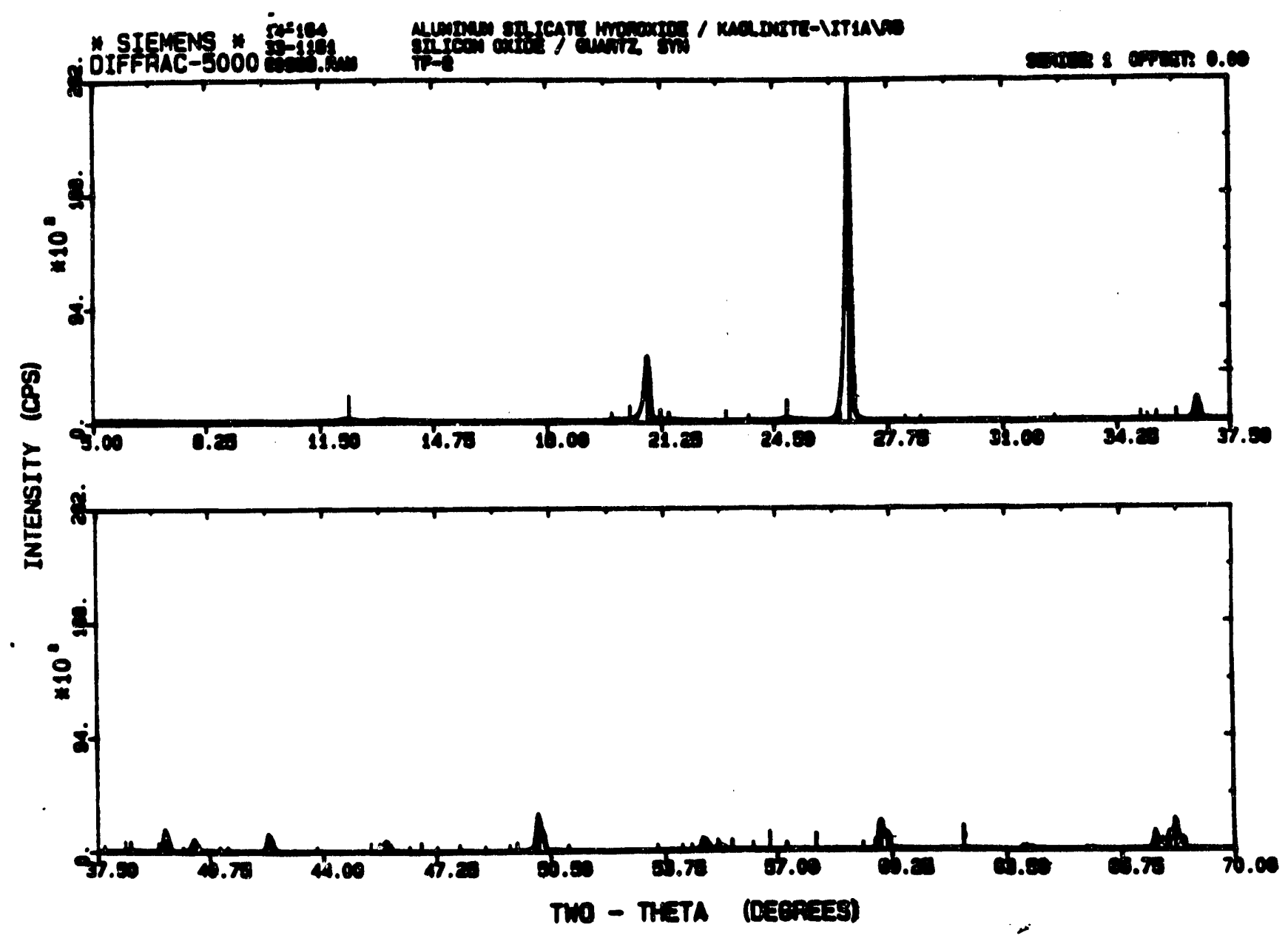




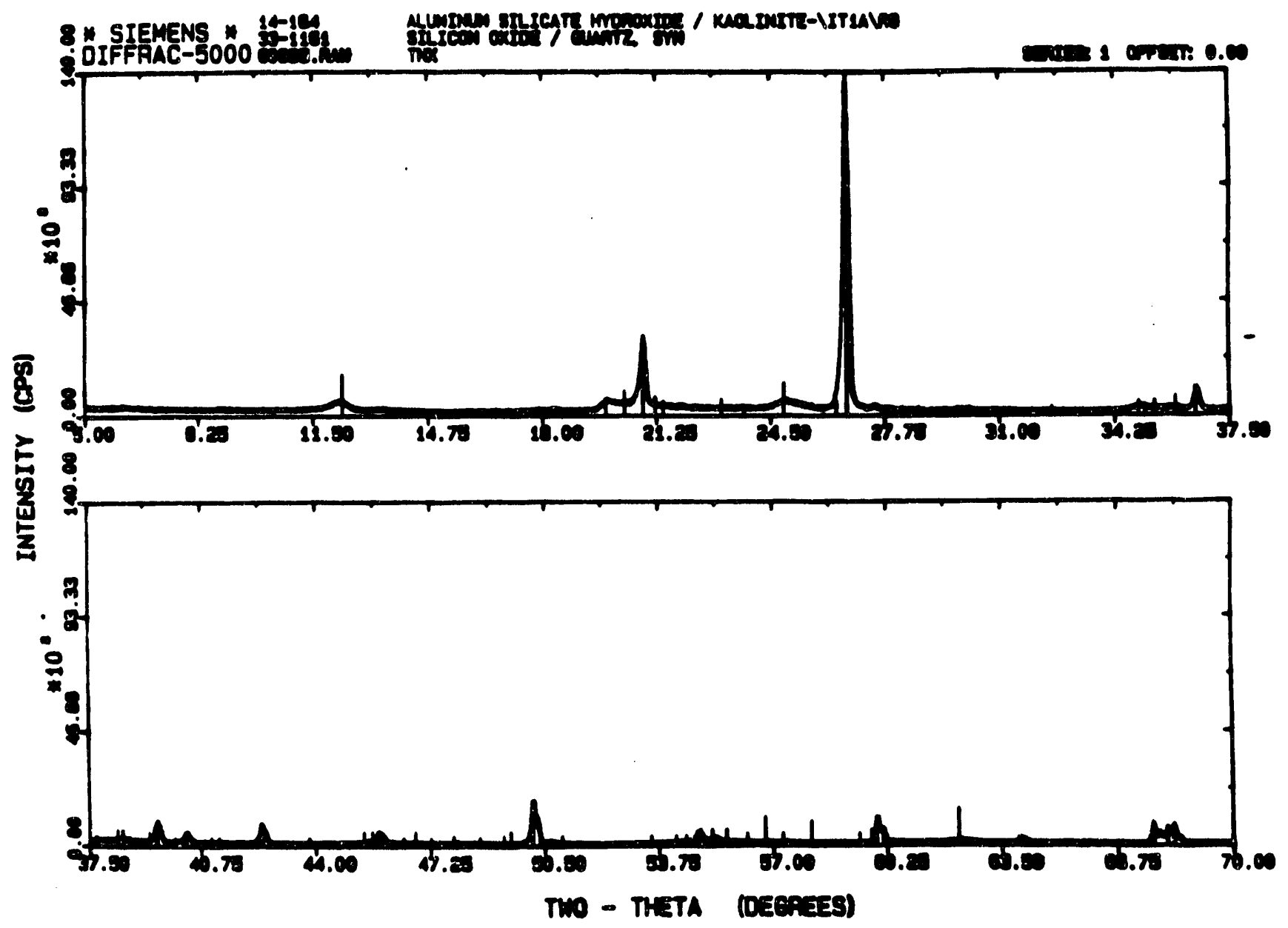


D.L. EISH

WSRC-RP-92-326

March 20, 1992

Page 44 of 45

SPECTRUM FOR ETE SOIL

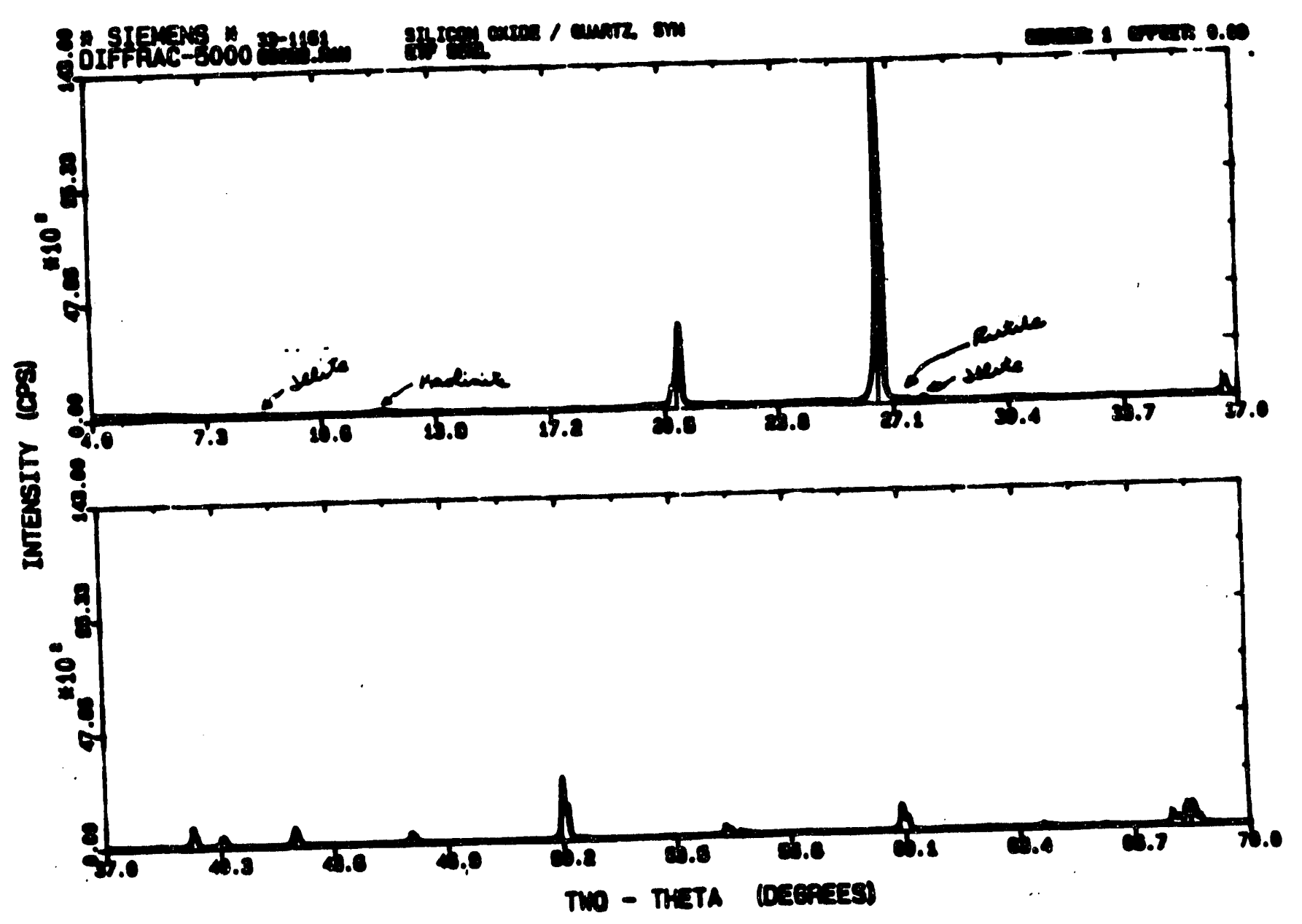


D.I. FISH

CC: W.L. Tamosaltis, 773-A

L.O. Dworjanyn, 779-2A

M.J. Barnes, 773-A

K.I. Dykes, 773-43A

S.C. Clark, SREL

R.D. Baena, Merrill-Lynch

A.D. Yu, 773-43A

J.Stuart Roberts, 241-84H (ETF)

CHC file, 773-A

SRL Records (4), 773-A
WSRC-RP- $92-326$

March 20, 1992

Page 45 of 45 
D.L. EISH

WSRC-RP-92-326

March 20, 1992

Page 44 of $45-44$

SPECTRUM EOR ETE SOIL

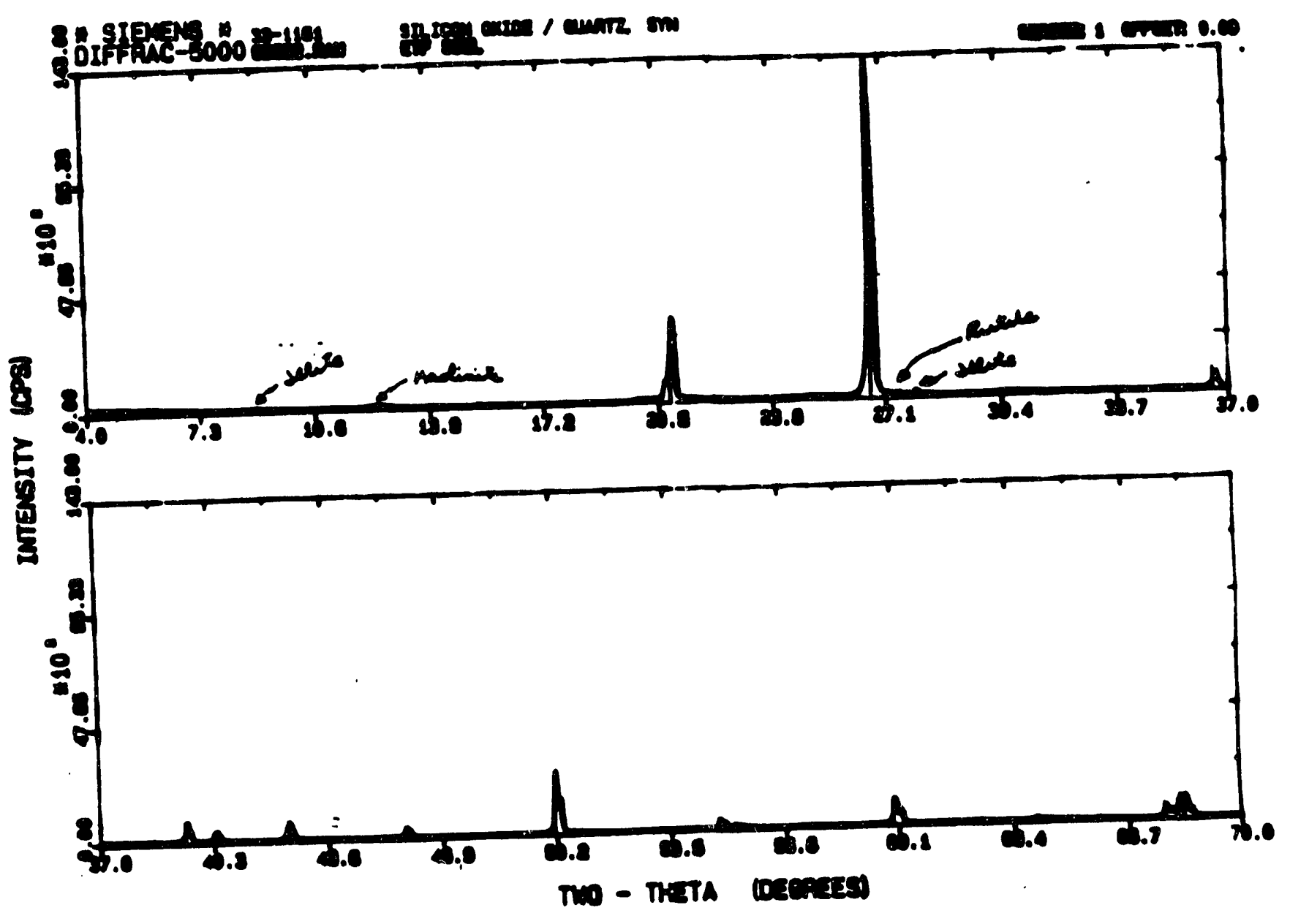



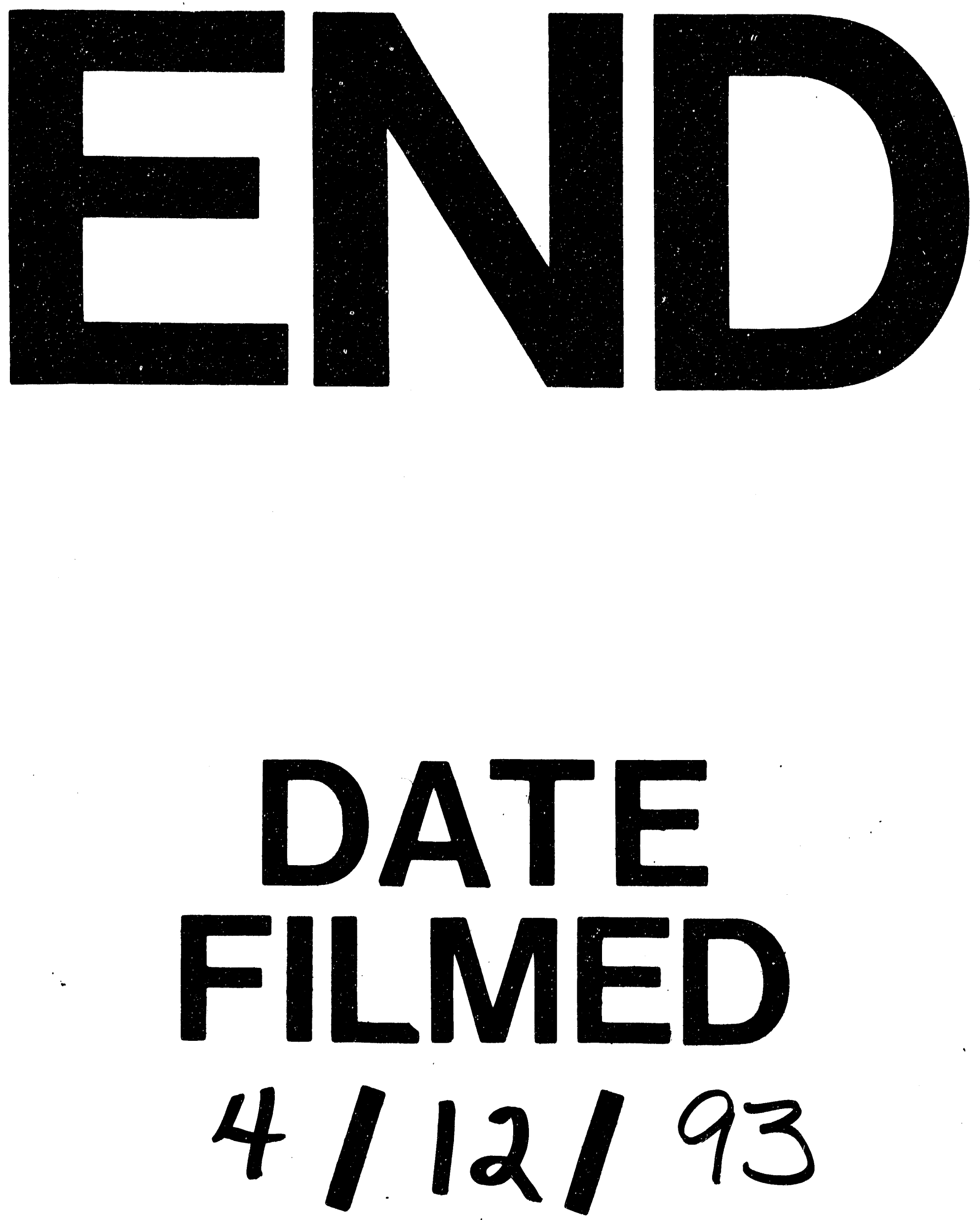
